\title{
Structure monoids of set-theoretic solutions of the Yang-Baxter equation*
}

\author{
Ferran Cedó $\quad$ Eric Jespers Charlotte Verwimp
}

\begin{abstract}
Given a set-theoretic solution $(X, r)$ of the Yang-Baxter equation, we denote by $M=M(X, r)$ the structure monoid and by $A=A(X, r)$, respectively $A^{\prime}=A^{\prime}(X, r)$, the left, respectively right, derived structure monoid of $(X, r)$. It is shown that there exist a left action of $M$ on $A$ and a right action of $M$ on $A^{\prime}$ and 1-cocycles $\pi$ and $\pi^{\prime}$ of $M$ with coefficients in $A$ and in $A^{\prime}$ with respect to these actions respectively. We investigate when the 1-cocycles are injective, surjective or bijective. In case $X$ is finite, it turns out that $\pi$ is bijective if and only if $(X, r)$ is left non-degenerate, and $\pi^{\prime}$ is bijective if and only if $(X, r)$ is right non-degenerate. In case $(X, r)$ is left non-degenerate, in particular $\pi$ is bijective, we define a semi-truss structure on $M(X, r)$ and then we show that this naturally induces a set-theoretic solution $(\bar{M}, \bar{r})$ on the least cancellative image $\bar{M}=M(X, r) / \eta$ of $M(X, r)$. In case $X$ is naturally embedded in $M(X, r) / \eta$, for example when $(X, r)$ is irretractable, then $\bar{r}$ is an extension of $r$. It also is shown that non-degenerate irretractable solutions necessarily are bijective.
\end{abstract}

2010 Mathematics Subject Classification: 16T25,20M05.

Key words: Yang-Baxter equation, Set-theoretic solution, Structure monoid, 1-cocycle, Semi-truss.

\section{Introduction}

Let $V$ be a vector space over a field $K$. Solutions $R: V \otimes V \rightarrow V \otimes V$ of the linear braid or Yang-Baxter equation (abbreviated YBE)

$$
\left(R \otimes \mathrm{id}_{V}\right) \circ\left(\mathrm{id}_{V} \otimes R\right) \circ\left(R \otimes \mathrm{id}_{V}\right)=\left(\mathrm{id}_{V} \otimes R\right) \circ\left(R \otimes \mathrm{id}_{V}\right) \circ\left(\mathrm{id}_{V} \otimes R\right)
$$

on the vector space $V \otimes V \otimes V$ have led to several algebraic structures, including some classes of bialgebras, quantum groups and Hopf algebras. Because the variety of solutions remains elusive, Drinfeld [11] in 1992 proposed to consider solutions that are linearizations of solutions on a basis of $V$, these are the so called set-theoretic solutions of the YBE. Thus a pair $(X, r)$, where $X$ is a non-empty set and $r: X \times X \rightarrow X \times X$ is a map, is called a set-theoretic solution of the YBE if

$$
\left(r \times \operatorname{id}_{X}\right) \circ\left(\operatorname{id}_{X} \times r\right) \circ\left(r \times \operatorname{id}_{X}\right)=\left(\operatorname{id}_{X} \times r\right) \circ\left(r \times \operatorname{id}_{X}\right) \circ\left(\operatorname{id}_{X} \times r\right) .
$$

For $x, y \in X$, write $r(x, y)=\left(\sigma_{x}(y), \gamma_{y}(x)\right)$. The solution $(X, r)$ is said to be left (resp. right) nondegenerate if each map $\sigma_{x}$ (resp. $\gamma_{y}$ ) is bijective. A left and right non-degenerate solution is simply called a non-degenerate solution. The solution $(X, r)$ is said to be involutive if $r^{2}=\operatorname{id}_{X \times X}$, in particular such a solution is bijective.

*The first author was partially supported by the grants MINECO-FEDER MTM2017-83487-P and AGAUR 2017SGR1725 (Spain). The second author is supported in part by Onderzoeksraad of Vrije Universiteit Brussel and Fonds voor Wetenschappelijk Onderzoek (Belgium). The third author is supported by Fonds voor Wetenschappelijk Onderzoek (Flanders), via an FWO Aspirant-mandate. 
This study started in the seminal papers of Etingof, Schedler and Soloviev 12 and Gateva-Ivanova and Van den Bergh [17. Since then, different aspects of this combinatorial problem have been developed 14, 17, 25, 26, 31 and several interesting connections have been found, such as braid and Garside groups [7, 9], (semi)groups of I-type [17, 21, matched pairs of groups [25, 32, Artin-Schelter regular algebras [13, Jacobson radical rings and generalizations [5, 27, regular subgroups and Hopf-Galois extensions [30, affine manifolds 28, orderability [3, 8, and factorizable groups 33 .

It is now well-known that all non-degenerate involutive set-theoretic solutions $(X, r)$ are restrictions of a set-theoretic solution on the structure monoid

$$
\left.M(X, r)=\langle x \in X| x y=\sigma_{x}(y) \gamma_{y}(x), \text { for all } x, y \in X\right\rangle .
$$

Furthermore, in this case, the structure group

$$
G(X, r)=\operatorname{gr}\left(x \in X \mid x y=\sigma_{x}(y) \gamma_{y}(x), \text { for all } x, y \in X\right)
$$

and the permutation group $\mathcal{G}(X, r)=\operatorname{gr}\left(\sigma_{x} \mid x \in X\right)$ have a brace structure, an algebraic structure introduced by Rump in [27. Moreover, in [2], it is shown that all finite non-degenerate involutive set-theoretic solutions with a given permutation group, as a brace, can be explicitly constructed. For this case of finite solutions $(X, r)$, Etingof, Schedler and Soloviev 12 proved that $G(X, r)$ is a finitely generated, solvable abelian-by-finite group and independently Gateva-Ivanova and Van den Bergh [17] have shown that $G(X, r)$ is a Bieberbach group, i.e. $G(X, r)$ is an abelian-by-finite group, torsion-free and finitely generated. To deal with arbitrary finite bijective non-degenerate solutions Guarnieri and Vendramin 18 introduced the algebraic structure called a skew brace. Bachiller [1] then also showed that all such solutions can be described from finite skew braces. Lu, Yan and Zhu [25, and Soloviev [31] showed that for such solutions the structure group $G(X, r)$ is abelian-by-finite (see also Lebed and Vendramin 24 for another proof), and Jespers, Kubat and Van Antwerpen [19] showed that the structure monoid $M(X, r)$ also is abelian-by-finite. Note that, if $(X, r)$ is not involutive then the canonical map $i: X \rightarrow G(X, r)$ is not necessarily injective and thus one cannot recover $r$ from the associated solution on $G(X, r)$, however it can be recovered from the solution associated to $M(X, r)$.

Also the associated structure algebras, i.e. the monoid algebra $K M(X, r)$ and the group algebra $K G(X, r)$, where $\mathrm{K}$ is any field, have been studied by Jespers and Okniński [21, Gateva-Ivanova and Van den Bergh [17. and Jespers, Kubat and Van Antwerpen [19]. In the latter it is shown that if $(X, r)$ is a left non-degenerate bijective finite set-theoretic solution then the algebra $K M(X, r)$ (and $K G(X, r)$ ) is a module-finite normal extension of a commutative affine subalgebra. In particular, these algebras are Noetherian PI-algebras of finite Gelfand-Kirillov dimension. Furthermore, it was shown that many properties, such as being a domain or prime, of the algebra $K M(X, r)$ are equivalent with the solution $(X, r)$ being involutive.

A crucial fact to prove the above results is (see [12, 19, 25]) that if $(X, r)$ is a left non-degenerate solution then the structure monoid $M(X, r)$ is a regular submonoid of the semidirect product

$$
A(X, r) \rtimes \mathcal{G}(X, r),
$$

where

$$
\left.A(X, r)=\langle x \in X| x \sigma_{x}(y)=\sigma_{x}(y) \sigma_{\sigma_{x}(y)}\left(\gamma_{y}(x)\right) \text {, for all } x, y \in X\right\rangle \text {, }
$$

i.e. for any element $a \in A(X, r)$ there is a unique $\phi(a) \in \mathcal{G}(X, r)$ such that $(a, \phi(a)) \in M(X, r)$. In particular, one has a bijective 1-cocycle $M(X, r) \rightarrow A(X, r)$, determined by the natural action of $\mathcal{G}(X, r)$ on $A(X, r)$. Here the derived monoid $A(X, r)$ "encodes" the relations determined by the map $r^{2}: X^{2} \rightarrow X^{2}$. If, furthermore, the left non-degenerate solution $(X, r)$ is bijective then the monoid $A=A(X, r)$ is such that $a A=A a$, for all $a \in A$. So $A(X, r)$ consists of normal elements and thus $A$ has a much richer structure than $M(X, r)$. For example, if $(X, r)$ is involutive then $A$ is a free abelian monoid of rank $|X|$. It is this "richer structure" that has been exploited in several papers to obtain information on the structure monoid $M(X, r)$ and the structure algebra $K M(X, r)$.

In this paper we continue these investigations for arbitrary set-theoretic solutions $(X, r)$. So, $r$ is not necessarily bijective and $X$ is any set. In the first section we recall the important result of Gateva-Ivanova 
and Majid [16: there exists a unique set-theoretic solution $\left(M, r_{M}\right)$ associated to the structure monoid $M=M(X, r)$ such that the restriction of $r_{M}$ to $X^{2}$ equals $r$. In the second section we introduce two derived monoids $A(X, r)$ and $A^{\prime}(X, r)$ and we prove that there is a unique 1-cocycle $\pi: M(X, r) \rightarrow A(X, r)$, with respect to the natural left action $\lambda^{\prime}: M(X, r) \rightarrow \operatorname{End}(A(X, r))$, such that $\pi(x)=x$, and a unique 1-cocycle $\pi^{\prime}: M(X, r) \rightarrow A^{\prime}(X, r)$, with respect to natural right action $\rho^{\prime}: M(X, r) \rightarrow \operatorname{End}\left(A^{\prime}(X, r)\right)$ such that $\pi^{\prime}(x)=x$. Hence one gets a monoid homomorphism $f: M(X, r) \rightarrow A(X, r) \rtimes \operatorname{Im}\left(\lambda^{\prime}\right): a \mapsto\left(\pi(a), \lambda_{a}^{\prime}\right)$ and a monoid anti-homomorphism $f^{\prime}: M(X, r) \rightarrow A^{\prime}(X, r)^{o p} \rtimes \operatorname{Im}\left(\rho^{\prime}\right): a \mapsto\left(\pi^{\prime}(a), \rho_{a}^{\prime}\right)$, where $\lambda_{x}^{\prime}(y)=\sigma_{x}(y)$ and $\rho_{x}^{\prime}(y)=\gamma_{x}(y)$, for all $x, y \in X$. In general these 1-cocycles are not bijective. But we investigate when they are respectively injective, respectively surjective. In case $(X, r)$ is finite the bijectiveness of $\pi$ (respectively $\pi^{\prime}$ ) is equivalent with the solution being left (respectively right) non-degenerate. In Section 4 we prove the surprising result that any non-degenerate irretractable solution is necessarily bijective. In Section 5 we link the algebraic structure of $M(X, r)$ to that of semi-trusses as introduced by Brzeziński [4. We determine the left cancellative (additive) congruence $\eta$ on $M(X, r)$ for $(X, r)$ a left non-degenerate solution, and we show that we obtain a solution $(M / \eta, \bar{r})$ determined by a semi-truss structure on $M / \eta$.

\section{Solution associated with the structure monoid}

In this section we recall a result of Gateva-Ivanova and Majid in [16, Section 3.2] stating that any set-theoretic solution $(X, r)$ of the YBE can be extended to a set-theoretic solution on its structure monoid $M(X, r)$. The result in [16 is stated for bijective solutions but the proof remains valid without this assumption.

Recall this construction. Let $(X, r)$ be a set-theoretic solution of the YBE which is not necessarily bijective. We write $r(x, y)=\left(\sigma_{x}(y), \gamma_{y}(x)\right)$, for all $x, y \in X$. It is known that $(X, r)$ is a set-theoretic solution of the YBE if and only if the following conditions hold:

$$
\begin{aligned}
\sigma_{x} \sigma_{y} & =\sigma_{\sigma_{x}(y)} \sigma_{\gamma_{y}(x)} \\
\sigma_{\gamma_{\sigma_{x}(y)}(z)}\left(\gamma_{y}(x)\right) & =\gamma_{\sigma_{\gamma_{x}(z)}(y)}\left(\sigma_{z}(x)\right) \\
\gamma_{x} \gamma_{y} & =\gamma_{\gamma_{x}(y)} \gamma_{\sigma_{y}(x)}
\end{aligned}
$$

for all $x, y, z \in X$.

Let $M=M(X, r)$ be the structure monoid of $(X, r)$, that is the multiplicative monoid with operation $\circ$ and with presentation

$$
\left.M(X, r)=\langle X| x \circ y=\sigma_{x}(y) \circ \gamma_{y}(x), \text { for all } x, y \in X\right\rangle .
$$

One defines the following "left action" on $M$ :

$$
\lambda: M \longrightarrow \operatorname{Map}(M, M): a \mapsto \lambda_{a},
$$

with $\lambda_{1}=\operatorname{id}_{M}$, and for $x_{1}, \ldots, x_{m}, y_{1}, \ldots, y_{n} \in X$ and $n>1, \lambda_{x_{1}}(1)=1$,

$$
\lambda_{x_{1}}\left(y_{1}\right)=\sigma_{x_{1}}\left(y_{1}\right), \quad \lambda_{x_{1}}\left(y_{1} \circ \cdots \circ y_{n}\right)=\sigma_{x_{1}}\left(y_{1}\right) \circ \lambda_{\gamma_{y_{1}}\left(x_{1}\right)}\left(y_{2} \circ \cdots \circ y_{n}\right),
$$

and for $m>1$,

$$
\lambda_{x_{1} \circ \cdots \circ x_{m}}=\lambda_{x_{1}} \circ \cdots \circ \lambda_{x_{m}} .
$$

One also defines a "right action" on $M$ :

$$
\rho: M \longrightarrow \operatorname{Map}(M, M): a \mapsto \rho_{a},
$$

with $\rho_{1}=\operatorname{id}_{M}$, and for $x_{1}, \ldots, x_{m}, y_{1}, \ldots, y_{n} \in X$ and $n>1$,

$$
\rho_{x_{1}}\left(y_{1}\right)=\gamma_{x_{1}}\left(y_{1}\right), \quad \rho_{x_{1}}\left(y_{1} \circ \cdots \circ y_{n}\right)=\rho_{\sigma_{y_{n}}\left(x_{1}\right)}\left(y_{1} \circ \cdots \circ y_{n-1}\right) \circ \gamma_{x_{1}}\left(y_{n}\right),
$$

and for $m>1$

$$
\rho_{x_{1} \circ \cdots \circ x_{m}}=\rho_{x_{m}} \circ \cdots \circ \rho_{x_{1}}
$$


In [16] it is proved that $\lambda$ and $\rho$ are well defined. Furthermore, it is then shown that every set-theoretic solution $(X, r)$ of the YBE is the restriction of a set-theoretic solution defined on the structure monoid $M(X, r)$.

Theorem 2.1 [16, Theorem 3.6] (Gateva-Ivanova and Majid) Let $(X, r)$ be a set-theoretic solution of the $Y B E$. Then the mapping $\lambda$ is a monoid homomorphism and the mapping $\rho$ is monoid anti-homomorphism such that

$$
\begin{aligned}
& \rho_{b}(c \circ a)=\rho_{\lambda_{a}(b)}(c) \circ \rho_{b}(a), \\
& \lambda_{b}(a \circ c)=\lambda_{b}(a) \circ \lambda_{\rho_{a}(b)}(c),
\end{aligned}
$$

for all $a, b, c \in M$. Furthermore, for $a, b \in M=M(X, r)$,

$$
a \circ b=\lambda_{a}(b) \circ \rho_{b}(a) .
$$

Let $r_{M}: M \times M \rightarrow M \times M$ be defined by $r_{M}(a, b)=\left(\lambda_{a}(b), \rho_{b}(a)\right)$, for all $a, b \in M$. Then, $\left(M, r_{M}\right)$ is $a$ set-theoretic solution of the YBE. Obviously, $r_{M}$ extends the solution $r$.

Note that if the solution $(X, r)$ is bijective and left and right non-degenerate, i.e. all $\sigma_{x}$ and $\gamma_{x}$ are bijective maps, then as in the proof of the above result one can show that the mappings $\sigma_{x}$ and $\gamma_{x}$ induce actually left and right actions on $G=G(X, r)$, say $\lambda^{e}: G \rightarrow \operatorname{Sym}(G)$ and $\rho^{e}: G \rightarrow \operatorname{Sym}(G)$; this is Theorem 4 in 25. Furthermore, the mapping $r_{G}(a, b)=\left(\lambda_{a}^{e}(b), \rho_{b}^{e}(a)\right)$, for $a, b \in G$, defines a set-theoretic solution on $G$. Note that, in general, the natural map $i: X \rightarrow G$ is not injective. One obtains that $r_{G}$ is an extension of the induced set-theoretic solution $\left(i(X), r_{i(X)^{2}}\right)=\left(i(X),\left(r_{G}\right)_{i(X)^{2}}\right)$.

A natural question is whether one can extend a solution $(X, r)$, via the actions induced from $\sigma_{x}$ and $\gamma_{y}$, to a solution on the structure group. This however is not possible in general as shown by the following example. Consider the set-theoretic solution $\left(X, \operatorname{id}_{X^{2}}\right)$ on a set $X$ with more than one element. Obviously, each $\sigma_{x}$ and $\gamma_{x}$ is the constant with image $\{x\}$. Hence, $M=M\left(X, \operatorname{id}_{X^{2}}\right)$ is the free monoid on the set $X$ and $G=G\left(X, \mathrm{id}_{X^{2}}\right)$ is the free group on $X$. However, because the maps $\sigma_{x}$ are not injective one cannot extend the maps $\sigma_{x}$ to a monoid homomorphism $\lambda: G \rightarrow \operatorname{Map}(G, G)$ with $\lambda_{x}(y)=\sigma_{x}(y)$, for $y \in G$.

A remarkable fact shown by Lu, Yan and Zhu in 25] is that if one can extend the mappings $\sigma_{x}$ and $\gamma_{x}$ to left and right actions on the structure group then the induced set-theoretic solution is bijective.

\section{Derived monoids}

Let $(X, r)$ be a set-theoretic solution of the YBE. Write $r(x, y)=\left(\sigma_{x}(y), \gamma_{y}(x)\right)$, for all $x, y \in X$. If $(X, r)$ is left non-degenerate, then Soloviev defined in [31] its derived solution $\left(X, r^{\prime}\right)$ by

$$
r^{\prime}(x, y)=\left(y, \sigma_{y} \gamma_{\sigma_{x}^{-1}(y)}(x)\right),
$$

for all $x, y \in X$. For general solutions one cannot define such a derived solution. But in [19] one defines the derived monoids of $(X, r)$ as

$$
\left.A(X, r)=\langle X| x+\sigma_{x}(y)=\sigma_{x}(y)+\sigma_{\sigma_{x}(y)} \gamma_{y}(x), \text { for all } x, y \in X\right\rangle
$$

and

$$
\left.A^{\prime}(X, r)=\langle X| \gamma_{y}(x) \oplus y=\gamma_{\gamma_{y}(x)} \sigma_{x}(y) \oplus \gamma_{y}(x) \text {, for all } x, y \in X\right\rangle .
$$

The zero element of $A(X, r)$ is denoted 0 and the zero element of $A^{\prime}(X, r)$ is denoted $0^{\prime}$. We will say that $A(X, r)$ is the left derived structure monoid of $(X, r)$ and $A^{\prime}(X, r)$ is the right derived structure monoid of $(X, r)$.

Note that $X \subseteq M(X, r), X \subseteq A(X, r)$ and $X \subseteq A^{\prime}(X, r)$, because the defining relations of these three monoids are homogeneous of degree 2 . 
Proposition 3.1 Let $(X, r)$ be a set-theoretic solution of the YBE, where $r(x, y)=\left(\sigma_{x}(y), \gamma_{y}(x)\right)$, for all $x, y \in X$. Then there exists a unique monoid homomorphism $\lambda^{\prime}: M(X, r) \longrightarrow \operatorname{End}(A(X, r))$ such that, $\lambda^{\prime}(x)(y)=\sigma_{x}(y)$, for all $x, y \in X$ and there exists a unique anti-homomorphism $\rho^{\prime}: M(X, r) \longrightarrow$ $\operatorname{End}\left(A^{\prime}(X, r)\right)$ such that, $\rho^{\prime}(x)(y)=\gamma_{x}(y)$, for all $x, y \in X$. Furthermore, if $(X, r)$ is left (right) nondegenerate, then $\operatorname{Im}\left(\lambda^{\prime}\right) \subseteq \operatorname{Aut}(A(X, r))\left(\operatorname{Im}\left(\rho^{\prime}\right) \subseteq \operatorname{Aut}\left(A^{\prime}(X, r)\right)\right)$.

Proof. We will write $\lambda^{\prime}(a)=\lambda_{a}^{\prime}$ and $\rho^{\prime}(a)=\rho_{a}^{\prime}$, for all $a \in M(X, r)$.

Let $x_{1}, \ldots, x_{m}, y_{1}, \ldots, y_{n} \in X$. We denote by $1,0,0^{\prime}$ the identity elements of the monoids $M(X, r)$, $A(X, r), A^{\prime}(X, r)$, respectively. We define $\lambda_{1}^{\prime}=\operatorname{id}_{A(X, r)}, \rho_{1}^{\prime}=\operatorname{id}_{A^{\prime}(X, r)}, \lambda_{a}^{\prime}(0)=0, \rho_{a}^{\prime}\left(0^{\prime}\right)=0^{\prime}$, for all $a \in M(X, r)$, and

$$
\lambda_{x_{1} \circ \cdots \circ x_{m}}^{\prime}\left(y_{1}+\cdots+y_{n}\right)=\sigma_{x_{1}} \ldots \sigma_{x_{m}}\left(y_{1}\right)+\cdots+\sigma_{x_{1}} \ldots \sigma_{x_{m}}\left(y_{n}\right)
$$

and

$$
\rho_{x_{1} \circ \cdots \circ x_{m}}^{\prime}\left(y_{1} \oplus \cdots \oplus y_{n}\right)=\gamma_{x_{m}} \ldots \gamma_{x_{1}}\left(y_{1}\right) \oplus \cdots \oplus \gamma_{x_{m}} \ldots \gamma_{x_{1}}\left(y_{n}\right)
$$

First we shall prove that $\lambda^{\prime}$ and $\rho^{\prime}$ are well-defined. To do so it is enough to prove that the following equalities hold:

$$
\begin{aligned}
\lambda_{x_{1} \circ x_{2}}^{\prime}\left(y_{1}+\cdots+y_{n}\right) & =\lambda_{\sigma_{x_{1}}\left(x_{2}\right) \circ \gamma_{x_{2}}\left(x_{1}\right)}^{\prime}\left(y_{1}+\cdots+y_{n}\right), \\
\lambda_{x_{1} \circ \cdots \circ x_{m}}^{\prime}\left(y_{1}+\sigma_{y_{1}}\left(y_{2}\right)\right) & =\lambda_{x_{1} \circ \cdots \circ x_{m}}^{\prime}\left(\sigma_{y_{1}}\left(y_{2}\right)+\sigma_{\sigma_{y_{1}}\left(y_{2}\right)}\left(\gamma_{y_{2}}\left(y_{1}\right)\right)\right), \\
\rho_{x_{1} \circ x_{2}}^{\prime}\left(y_{1} \oplus \cdots \oplus y_{n}\right) & =\rho_{\sigma_{x_{1}}\left(x_{2}\right) \circ \gamma_{x_{2}}\left(x_{1}\right)}^{\prime}\left(y_{1} \oplus \cdots \oplus y_{n}\right), \\
\rho_{x_{1} \circ \cdots \circ x_{m}}^{\prime}\left(\gamma_{y_{2}}\left(y_{1}\right) \oplus y_{2}\right) & =\rho_{x_{1} \circ \cdots \circ x_{m}}^{\prime}\left(\gamma_{\gamma_{y_{2}}\left(y_{1}\right)}\left(\sigma_{y_{1}}\left(y_{2}\right) \oplus \oplus \gamma_{y_{2}}\left(y_{1}\right)\right) .\right.
\end{aligned}
$$

Using the relations (11) and (3), equations (11) and (13) are easily checked:

$$
\begin{aligned}
\lambda_{x_{1} \circ x_{2}}^{\prime}\left(y_{1}+\cdots+y_{n}\right) & =\sigma_{x_{1}} \sigma_{x_{2}}\left(y_{1}\right)+\cdots+\sigma_{x_{1}} \sigma_{x_{2}}\left(y_{n}\right) \\
& =\sigma_{\sigma_{x_{1}}\left(x_{2}\right)} \sigma_{\gamma_{x_{2}}\left(x_{1}\right)}\left(y_{1}\right)+\cdots+\sigma_{\sigma_{x_{1}}\left(x_{2}\right)} \sigma_{\gamma_{x_{2}}\left(x_{1}\right)}\left(y_{n}\right) \\
& =\lambda_{\sigma_{x_{1}}\left(x_{2}\right) \circ \gamma_{x_{2}}\left(x_{1}\right)}^{\prime}\left(y_{1}+\cdots+y_{n}\right), \\
\rho_{x_{1} \circ x_{2}}^{\prime}\left(y_{1} \oplus \cdots \oplus y_{n}\right) & =\gamma_{x_{2}} \gamma_{x_{1}}\left(y_{1}\right) \oplus \cdots \oplus \gamma_{x_{2}} \gamma_{x_{1}}\left(y_{n}\right) \\
& =\gamma_{\gamma_{x_{2}}\left(x_{1}\right)} \gamma_{\sigma_{x_{1}}\left(x_{2}\right)}\left(y_{1}\right) \oplus \cdots \oplus \gamma_{\gamma_{x_{2}}\left(x_{1}\right)} \gamma_{\sigma_{x_{1}}\left(x_{2}\right)}\left(y_{n}\right) \\
& =\rho_{\sigma_{x_{1}}\left(x_{2}\right) \circ \gamma_{x_{2}}\left(x_{1}\right)}^{\prime}\left(y_{1} \oplus \cdots \oplus y_{n}\right) .
\end{aligned}
$$

Using the relations (11), (21) and (3) we shall prove equations (12) and (14) by induction on $m$. For $m=0$, (12) and (14) follows by the defining relations of $A(X, r)$ and $A^{\prime}(X, r)$. Suppose that $m>0$. Assume that $\lambda_{x_{1} \circ \cdots \circ x_{k}}^{\prime}\left(y_{1}+\sigma_{y_{1}}\left(y_{2}\right)\right)=\lambda_{x_{1} \circ \cdots \circ x_{k}}^{\prime}\left(\sigma_{y_{1}}\left(y_{2}\right)+\sigma_{\sigma_{y_{1}}\left(y_{2}\right)}\left(\gamma_{y_{2}}\left(y_{1}\right)\right)\right)$ and $\rho_{x_{1} \circ \cdots \circ x_{k}}^{\prime}\left(\gamma_{y_{2}}\left(y_{1}\right) \oplus y_{2}\right)=$ $\rho_{x_{1} \circ \cdots \circ x_{k}}^{\prime}\left(\gamma_{\gamma_{y_{2}}\left(y_{1}\right)}\left(\sigma_{y_{1}}\left(y_{2}\right)\right) \oplus \gamma_{y_{2}}\left(y_{1}\right)\right)$, for $k<m$, then

$$
\begin{aligned}
& \lambda_{x_{1} \circ \cdots \circ x_{m}}^{\prime}\left(y_{1}+\sigma_{y_{1}}\left(y_{2}\right)\right) \\
& =\sigma_{x_{1}} \cdots \sigma_{x_{m}}\left(y_{1}\right)+\sigma_{x_{1}} \cdots \sigma_{x_{m}}\left(\sigma_{y_{1}}\left(y_{2}\right)\right) \\
& =\lambda_{x_{1} 0 \cdots \circ x_{m-1}}^{\prime}\left(\sigma_{x_{m}}\left(y_{1}\right)+\sigma_{x_{m}}\left(\sigma_{y_{1}}\left(y_{2}\right)\right)\right) \\
& =\lambda_{x_{1} \circ \cdots \circ x_{m-1}}^{\prime}\left(\sigma_{x_{m}}\left(y_{1}\right)+\sigma_{\sigma_{x_{m}}\left(y_{1}\right)}\left(\sigma_{\gamma_{y_{1}}\left(x_{m}\right)}\left(y_{2}\right)\right)\right) \\
& =\lambda_{x_{1} \circ \cdots \circ x_{m-1}}^{\prime}\left(\sigma_{\sigma_{x_{m}}\left(y_{1}\right)}\left(\sigma_{\gamma_{y_{1}}\left(x_{m}\right)}\left(y_{2}\right)\right)+\sigma_{\sigma_{\sigma_{x_{m}}\left(y_{1}\right)}\left(\sigma_{\gamma_{y_{1}}\left(x_{m}\right)}\left(y_{2}\right)\right)}\left(\gamma_{\sigma_{\gamma_{y_{1}}\left(x_{m}\right)}\left(y_{2}\right)}\left(\sigma_{x_{m}}\left(y_{1}\right)\right)\right)\right) \\
& =\lambda_{x_{1} \circ \cdots \circ x_{m-1}}^{\prime}\left(\sigma_{x_{m}}\left(\sigma_{y_{1}}\left(y_{2}\right)\right)+\sigma_{\sigma_{x_{m}}\left(\sigma_{y_{1}}\left(y_{2}\right)\right)}\left(\gamma_{\sigma_{\gamma_{y_{1}}\left(x_{m}\right)}\left(y_{2}\right)}\left(\sigma_{x_{m}}\left(y_{1}\right)\right)\right)\right) \\
& =\lambda_{x_{1} \circ \cdots \circ x_{m-1}}^{\prime}\left(\sigma_{x_{m}}\left(\sigma_{y_{1}}\left(y_{2}\right)\right)+\sigma_{\sigma_{x_{m}}\left(\sigma_{y_{1}}\left(y_{2}\right)\right)}\left(\sigma_{\gamma_{\sigma_{y_{1}}\left(y_{2}\right)}\left(x_{m}\right)}\left(\gamma_{y_{2}}\left(y_{1}\right)\right)\right)\right) \\
& =\lambda_{x_{1} \circ \cdots \circ x_{m-1}}^{\prime}\left(\sigma_{x_{m}}\left(\sigma_{y_{1}}\left(y_{2}\right)\right)+\sigma_{x_{m}}\left(\sigma_{\sigma_{y_{1}}\left(y_{2}\right)}\left(\gamma_{y_{2}}\left(y_{1}\right)\right)\right)\right) \\
& =\sigma_{x_{1}} \cdots \sigma_{x_{m}}\left(\sigma_{y_{1}}\left(y_{2}\right)\right)+\sigma_{x_{1}} \cdots \sigma_{x_{m}}\left(\sigma_{\sigma_{y_{1}}\left(y_{2}\right)}\left(\gamma_{y_{2}}\left(y_{1}\right)\right)\right) \\
& =\lambda_{x_{1} \circ \cdots \circ x_{m}}^{\prime}\left(\sigma_{y_{1}}\left(y_{2}\right)+\sigma_{\sigma_{y_{1}}\left(y_{2}\right)}\left(\gamma_{y_{2}}\left(y_{1}\right)\right)\right),
\end{aligned}
$$


and

$$
\begin{aligned}
& \rho_{x_{1} \circ \cdots \circ x_{m}}^{\prime}\left(\gamma_{y_{2}}\left(y_{1}\right) \oplus y_{2}\right) \\
& =\rho_{x_{2} \circ \cdots \circ x_{m}}^{\prime}\left(\gamma_{x_{1}}\left(\gamma_{y_{2}}\left(y_{1}\right)\right) \oplus \gamma_{x_{1}}\left(y_{2}\right)\right) \\
& =\rho_{x_{2} \circ \cdots \circ x_{m}}^{\prime}\left(\gamma_{\gamma_{x_{1}}\left(y_{2}\right)}\left(\gamma_{\sigma_{y_{2}}\left(x_{1}\right)}\left(y_{1}\right)\right) \oplus \gamma_{x_{1}}\left(y_{2}\right)\right) \\
& =\rho_{x_{x^{2}} \cdots \circ x_{m}}^{\prime}\left(\gamma_{\gamma_{\gamma_{x_{1}}\left(y_{2}\right)}\left(\gamma_{\sigma_{y_{2}}\left(x_{1}\right)}\left(y_{1}\right)\right)}\left(\sigma_{\gamma_{\sigma_{y_{2}}\left(x_{1}\right)}\left(y_{1}\right)}\left(\gamma_{x_{1}}\left(y_{2}\right)\right)\right) \oplus \gamma_{\gamma_{x_{1}}\left(y_{2}\right)}\left(\gamma_{\sigma_{y_{2}}\left(x_{1}\right)}\left(y_{1}\right)\right)\right) \\
& =\rho_{x_{2} \circ \cdots \circ x_{m}}^{\prime}\left(\gamma_{\gamma_{x_{1}}\left(\gamma_{y_{2}}\left(y_{1}\right)\right)}\left(\sigma_{\gamma_{\sigma_{y_{2}}\left(x_{1}\right)}\left(y_{1}\right)}\left(\gamma_{x_{1}}\left(y_{2}\right)\right)\right) \oplus \gamma_{x_{1}}\left(\gamma_{y_{2}}\left(y_{1}\right)\right)\right) \\
& =\rho_{x_{2} \circ \cdots \circ x_{m}}^{\prime}\left(\gamma_{\gamma_{x_{1}}\left(\gamma_{y_{2}}\left(y_{1}\right)\right)}\left(\gamma_{\sigma_{\gamma_{y_{2}}\left(y_{1}\right)}\left(x_{1}\right)}\left(\sigma_{y_{1}}\left(y_{2}\right)\right) \oplus \gamma_{x_{1}}\left(\gamma_{y_{2}}\left(y_{1}\right)\right)\right)\right. \\
& =\rho_{x_{2} \circ \cdots \circ x_{m}}^{\prime}\left(\gamma_{x_{1}}\left(\gamma_{\gamma_{y_{2}}\left(y_{1}\right)}\left(\sigma_{y_{1}}\left(y_{2}\right)\right)\right) \oplus \gamma_{x_{1}}\left(\gamma_{y_{2}}\left(y_{1}\right)\right)\right) \\
& =\gamma_{x_{m}} \cdots \gamma_{x_{1}}\left(\gamma_{\gamma_{y_{2}}\left(y_{1}\right)}\left(\sigma_{y_{1}}\left(y_{2}\right)\right)\right) \oplus \gamma_{x_{m}} \cdots \gamma_{x_{1}}\left(\gamma_{y_{2}}\left(y_{1}\right)\right) \\
& =\rho_{x_{1} \circ \cdots \circ x_{m}}^{\prime}\left(\gamma_{\gamma_{y_{2}}\left(y_{1}\right)}\left(\sigma_{y_{1}}\left(y_{2}\right)\right) \oplus \gamma_{y_{2}}\left(y_{1}\right)\right)
\end{aligned}
$$

This proves that $\lambda_{a}^{\prime}$ and $\rho_{a}^{\prime}$ are well-defined and clearly $\lambda_{a}^{\prime} \in \operatorname{End}(A(X, r))$ and $\rho_{a}^{\prime} \in \operatorname{End}\left(A^{\prime}(X, r)\right)$, for all $a \in M(X, r)$. Thus $\lambda^{\prime}$ and $\rho^{\prime}$ are well-defined. It is clear that $\lambda^{\prime}$ is a monoid homomorphism and that it is unique with respect to the condition $\lambda_{x}^{\prime}(y)=\sigma_{x}(y)$, for all $x, y \in X$. It also is clear that $\rho^{\prime}$ is a monoid anti-homomorphism and that it is unique for the condition $\rho_{x}^{\prime}(y)=\gamma_{x}(y)$, for all $x, y \in X$.

Assume now that $(X, r)$ is left non-degenerate. Let $x, y_{1}, \ldots, y_{n} \in X$. We define $f_{x} \in \operatorname{End}(A(X, r))$ by

$$
f_{x}\left(y_{1}+\cdots+y_{n}\right)=\sigma_{x}^{-1}\left(y_{1}\right)+\cdots+\sigma_{x}^{-1}\left(y_{n}\right) .
$$

To see that $f_{x}$ is well-defined it is enough to prove that

$$
f_{x}\left(y_{1}+\sigma_{y_{1}}\left(y_{2}\right)\right)=f_{x}\left(\sigma_{y_{1}}\left(y_{2}\right)+\sigma_{\sigma_{y_{1}}\left(y_{2}\right)}\left(\gamma_{y_{2}}\left(y_{1}\right)\right)\right) .
$$

Note that, from (1),

$$
\sigma_{x}^{-1} \sigma_{y_{1}}\left(y_{2}\right)=\sigma_{\sigma_{x}^{-1}\left(y_{1}\right)} \sigma_{\gamma_{\sigma_{x}^{-1}\left(y_{1}\right)}^{-1}(x)}\left(y_{2}\right)
$$

and thus, also using (2), we get that

$$
\begin{aligned}
& \sigma_{\gamma_{\sigma_{x}^{-1} \sigma_{y_{1}\left(y_{2}\right)}(x)}} \gamma_{\sigma_{\sigma_{\sigma_{x}^{-1}\left(y_{1}\right)}^{-1}(x)}\left(y_{2}\right)}\left(\sigma_{x}^{-1}\left(y_{1}\right)\right) \\
& =\sigma_{\gamma_{\sigma_{x}^{-1}\left(y_{1}\right)} \sigma_{\gamma_{\sigma_{x}^{-1}\left(y_{1}\right)}^{-1}(x)}\left(y_{2}\right)}(x) \gamma_{\sigma_{\sigma_{x}^{-1}\left(y_{1}\right)}^{-1}\left(x^{\prime}\left(y_{2}\right)\right.}\left(\sigma_{x}^{-1}\left(y_{1}\right)\right) \\
& =\gamma_{\sigma_{\sigma_{x}^{-1}\left(y_{1}\right)}(x)}\left(\sigma_{\gamma_{\sigma_{x}^{-1}\left(y_{1}\right)}^{-1}(x)}\left(y_{2}\right)\right) \sigma_{x}\left(\sigma_{x}^{-1}\left(y_{1}\right)\right) \\
& =\gamma_{y_{2}}\left(y_{1}\right) \text {. }
\end{aligned}
$$

We have that

$$
\begin{aligned}
& f_{x}\left(y_{1}+\sigma_{y_{1}}\left(y_{2}\right)\right) \\
& =\sigma_{x}^{-1}\left(y_{1}\right)+\sigma_{x}^{-1}\left(\sigma_{y_{1}}\left(y_{2}\right)\right) \\
& \stackrel{15}{=} \sigma_{x}^{-1}\left(y_{1}\right)+\sigma_{\sigma_{x}^{-1}\left(y_{1}\right)}\left(\sigma_{\gamma_{\sigma_{x}^{-1}\left(y_{1}\right)}^{-1}(x)}^{-1}\left(y_{2}\right)\right) \\
& =\sigma_{\sigma_{x}^{-1}\left(y_{1}\right)}\left(\sigma_{\sigma_{\sigma_{x}^{-1}\left(y_{1}\right)}^{-1}(x)}\left(y_{2}\right)\right)+\sigma_{\sigma_{\sigma_{x}^{-1}\left(y_{1}\right)}\left(\sigma_{\sigma_{\sigma_{x}^{-1}\left(y_{1}\right)}^{-1}}\left(x^{-1}\right)\right)}\left(\gamma_{\sigma_{\sigma_{x}^{-1}\left(y_{1}\right)}^{-1}(x)}\left(y_{2}\right)\right. \\
& \stackrel{15}{=} \sigma_{x}^{-1}\left(\sigma_{y_{1}}^{-1}\left(y_{2}\right)\right)+\sigma_{\sigma_{x}^{-1}\left(\sigma_{y_{1}}\left(y_{2}\right)\right)}\left(\gamma_{\sigma_{\left.\sigma_{\sigma_{x}^{-1}\left(y_{1}\right)}^{-1}\right)}\left(y_{2}\right)}\left(\sigma_{x}^{-1}\left(y_{1}\right)\right)\right) \\
& \stackrel{16}{=} \sigma_{x}^{-1}\left(\sigma_{y_{1}}\left(y_{2}\right)\right)+\sigma_{\sigma_{x}^{-1}\left(\sigma_{y_{1}}\left(y_{2}\right)\right)}\left(\sigma_{\gamma_{\sigma_{x}^{-1} \sigma_{y_{1}\left(y_{2}\right)}}^{-1}(x)}\left(\gamma_{y_{2}}\left(y_{1}\right)\right)\right)
\end{aligned}
$$




$$
\begin{aligned}
& \stackrel{15}{=} \sigma_{x}^{-1}\left(\sigma_{y_{1}}\left(y_{2}\right)\right)+\sigma_{x}^{-1}\left(\sigma_{\sigma_{y_{1}}\left(y_{2}\right)}\left(\gamma_{y_{2}}\left(y_{1}\right)\right)\right) \\
& =f_{x}\left(\sigma_{y_{1}}\left(y_{2}\right)+\sigma_{\sigma_{y_{1}}\left(y_{2}\right)}\left(\gamma_{y_{2}}\left(y_{1}\right)\right)\right)
\end{aligned}
$$

where the third equality follows from the defining relations in $A(X, r)$. Hence $f_{x}$ is well-defined. Note that $f_{x} \lambda_{x}^{\prime}=\lambda_{x}^{\prime} f_{x}=$ id. Thus $\lambda_{x}^{\prime} \in \operatorname{Aut}(A(X, r))$, for all $x \in X$. Therefore $\operatorname{Im}\left(\lambda^{\prime}\right) \subseteq \operatorname{Aut}(A(X, r))$.

Similarly one can prove that if $(X, r)$ is right non-degenerate, then $\operatorname{Im}\left(\rho^{\prime}\right) \subseteq \operatorname{Aut}\left(A^{\prime}(X, r)\right)$.

Proposition 3.2 Let $(X, r)$ be a set-theoretic solution of the YBE. Then

(i) there is a unique 1-cocycle $\pi: M(X, r) \longrightarrow A(X, r)$ with respect to the left action $\lambda^{\prime}$ such that $\pi(x)=x$, for all $x \in X$.

(ii) there is a unique 1-cocycle $\pi^{\prime}: M(X, r) \longrightarrow A^{\prime}(X, r)$ with respect to the right action $\rho^{\prime}$ such that $\pi^{\prime}(x)=x$, for all $x \in X$.

Furthermore, the mapping

$$
f: M(X, r) \rightarrow A(X, r) \rtimes \operatorname{Im}\left(\lambda^{\prime}\right): a \mapsto\left(\pi(a), \lambda_{a}^{\prime}\right)
$$

is a monoid homomorphism and the mapping

$$
f^{\prime}: M(X, r) \rightarrow A^{\prime}(X, r)^{o p} \rtimes \operatorname{Im}\left(\rho^{\prime}\right): a \mapsto\left(\pi^{\prime}(a), \rho_{a}^{\prime}\right)
$$

is a monoid anti-homomorphism.

Proof. We define for $x_{1}, \ldots, x_{m} \in X$,

$$
\begin{aligned}
\pi(1) & =0, \\
\pi\left(x_{1}\right) & =x_{1}, \quad \text { and for } m>1, \\
\pi\left(x_{1} \circ \cdots \circ x_{m}\right) & =x_{1}+\lambda_{x_{1}}^{\prime}\left(\pi\left(x_{2} \circ \cdots \circ x_{m}\right)\right), \\
\pi^{\prime}(1) & =0^{\prime}, \\
\pi^{\prime}\left(x_{1}\right) & =x_{1}, \quad \text { and for } m>1, \\
\pi^{\prime}\left(x_{1} \circ \cdots \circ x_{m}\right) & =\rho_{x_{m}}^{\prime}\left(\pi^{\prime}\left(x_{1} \circ \cdots \circ x_{m-1}\right)\right) \oplus x_{m} .
\end{aligned}
$$

We prove that $\pi\left(x_{1} \circ \cdots \circ x_{m}\right)$ and $\pi^{\prime}\left(x_{1} \circ \cdots \circ x_{m}\right)$ are well-defined by induction on $m$. For $m=1$ it is clear. Suppose that $m>1$ and that $\pi\left(x_{1} \circ \cdots \circ x_{m-1}\right)$ and $\pi^{\prime}\left(x_{1} \circ \cdots \circ x_{m-1}\right)$ are well-defined.

By the induction hypothesis, it is enough to show that

$$
x_{1}+\lambda_{x_{1}}^{\prime}\left(\pi\left(x_{2} \circ \cdots \circ x_{m}\right)\right)=\sigma_{x_{1}}\left(x_{2}\right)+\lambda_{\sigma_{x_{1}}\left(x_{2}\right)}^{\prime}\left(\pi\left(\gamma_{x_{2}}\left(x_{1}\right) \circ x_{3} \circ \cdots \circ x_{m}\right)\right)
$$

and

$$
\rho_{x_{m}}^{\prime}\left(\pi^{\prime}\left(x_{1} \circ \cdots \circ x_{m-1}\right)\right) \oplus x_{m}=\rho_{\gamma_{x_{m}}\left(x_{m-1}\right)}^{\prime}\left(\pi^{\prime}\left(x_{1} \circ \cdots \circ x_{m-2} \circ \sigma_{x_{m-1}}\left(x_{m}\right)\right)\right) \oplus \gamma_{x_{m}}\left(x_{m-1}\right) .
$$

By (11) and (13) we get that

$$
\begin{aligned}
& \sigma_{x_{1}}\left(x_{2}\right)+\lambda_{\sigma_{x_{1}}\left(x_{2}\right)}^{\prime}\left(\pi\left(\gamma_{x_{2}}\left(x_{1}\right) \circ x_{3} \circ \cdots \circ x_{m}\right)\right) \\
& \quad=\sigma_{x_{1}}\left(x_{2}\right)+\lambda_{\sigma_{x_{1}}\left(x_{2}\right)}^{\prime}\left(\gamma_{x_{2}}\left(x_{1}\right)+\lambda_{\gamma_{x_{2}}\left(x_{1}\right)}^{\prime}\left(\pi\left(x_{3} \circ \cdots \circ x_{m}\right)\right)\right) \\
& \quad=\sigma_{x_{1}}\left(x_{2}\right)+\sigma_{\sigma_{x_{1}}\left(x_{2}\right)}\left(\gamma_{x_{2}}\left(x_{1}\right)\right)+\lambda_{\sigma_{x_{1}}\left(x_{2}\right)}^{\prime}\left(\lambda_{\gamma_{x_{2}}\left(x_{1}\right)}^{\prime}\left(\pi\left(x_{3} \circ \cdots \circ x_{m}\right)\right)\right) \\
& \quad=x_{1}+\sigma_{x_{1}}\left(x_{2}\right)+\lambda_{\sigma_{x_{1}}\left(x_{2}\right) \circ \gamma_{x_{2}}\left(x_{1}\right)}^{\prime}\left(\pi\left(x_{3} \circ \cdots \circ x_{m}\right)\right) \\
& =x_{1}+\sigma_{x_{1}}\left(x_{2}\right)+\lambda_{x_{1} \circ x_{2}}^{\prime}\left(\pi\left(x_{3} \circ \cdots \circ x_{m}\right)\right) \\
& =x_{1}+\sigma_{x_{1}}\left(x_{2}\right)+\lambda_{x_{1}}^{\prime}\left(\lambda_{x_{2}}^{\prime}\left(\pi\left(x_{3} \circ \cdots \circ x_{m}\right)\right)\right) \\
& =x_{1}+\lambda_{x_{1}}^{\prime}\left(x_{2}+\lambda_{x_{2}}^{\prime}\left(\pi\left(x_{3} \circ \cdots \circ x_{m}\right)\right)\right) \\
& =x_{1}+\lambda_{x_{1}}^{\prime}\left(\pi\left(x_{2} \circ \cdots \circ x_{m}\right)\right)
\end{aligned}
$$


and

$$
\begin{aligned}
& \rho_{\gamma_{x_{m}}\left(x_{m-1}\right)}^{\prime}\left(\pi^{\prime}\left(x_{1} \circ \cdots \circ x_{m-2} \circ \sigma_{x_{m-1}}\left(x_{m}\right)\right)\right) \oplus \gamma_{x_{m}}\left(x_{m-1}\right) \\
& \quad=\rho_{\gamma_{x_{m}}\left(x_{m-1}\right)}^{\prime}\left(\rho_{\sigma_{x_{m-1}}\left(x_{m}\right)}^{\prime}\left(\pi^{\prime}\left(x_{1} \circ \cdots \circ x_{m-2}\right)\right) \oplus \sigma_{x_{m-1}}\left(x_{m}\right)\right) \oplus \gamma_{x_{m}}\left(x_{m-1}\right) \\
& \quad=\rho_{\gamma_{x_{m}}\left(x_{m-1}\right)}^{\prime}\left(\rho_{\sigma_{x_{m-1}}\left(x_{m}\right)}^{\prime}\left(\pi^{\prime}\left(x_{1} \circ \cdots \circ x_{m-2}\right)\right)\right) \oplus \gamma_{\gamma_{x_{m}}\left(x_{m-1}\right)}\left(\sigma_{x_{m-1}}\left(x_{m}\right)\right) \oplus \gamma_{x_{m}}\left(x_{m-1}\right) \\
& \quad=\rho_{\sigma_{x_{m-1}}\left(x_{m}\right) \circ \gamma_{x_{m}}\left(x_{m-1}\right)}^{\prime}\left(\pi^{\prime}\left(x_{1} \circ \cdots \circ x_{m-2}\right)\right) \oplus \gamma_{x_{m}}\left(x_{m-1}\right) \oplus x_{m} \\
& =\rho_{x_{m-1} \circ x_{m}}^{\prime}\left(\pi^{\prime}\left(x_{1} \circ \cdots \circ x_{m-2}\right)\right) \oplus \gamma_{x_{m}}\left(x_{m-1}\right) \oplus x_{m} \\
& =\rho_{x_{m}}^{\prime}\left(\rho_{x_{m-1}}^{\prime}\left(\pi^{\prime}\left(x_{1} \circ \cdots \circ x_{m-2}\right)\right)\right) \oplus \gamma_{x_{m}}\left(x_{m-1}\right) \oplus x_{m} \\
& =\rho_{x_{m}}^{\prime}\left(\rho_{x_{m-1}}^{\prime}\left(\pi^{\prime}\left(x_{1} \circ \cdots \circ x_{m-2}\right)\right) \oplus x_{m-1}\right) \oplus x_{m} \\
& =\rho_{x_{m}}^{\prime}\left(\pi^{\prime}\left(x_{1} \circ \cdots \circ x_{m-1}\right)\right) \oplus x_{m} .
\end{aligned}
$$

Thus, indeed, $\pi$ and $\pi^{\prime}$ are well-defined.

For all $a, b \in M(X, r)$, we shall prove by induction on $\operatorname{deg}(a)+\operatorname{deg}(b)$ that

$$
\pi(a \circ b)=\pi(a)+\lambda_{a}^{\prime}(\pi(b))
$$

and

$$
\pi^{\prime}(a \circ b)=\rho_{b}^{\prime}\left(\pi^{\prime}(a)\right) \oplus \pi^{\prime}(b) .
$$

If $\operatorname{deg}(a)=\operatorname{deg}(b)=1$, then (19) and (20) follow by definition. Hence, we may suppose that $\operatorname{deg}(a)+\operatorname{deg}(b)>$ 2 and that $\pi\left(a^{\prime} \circ b^{\prime}\right)=\pi\left(a^{\prime}\right)+\lambda_{a^{\prime}}^{\prime}\left(\pi\left(b^{\prime}\right)\right)$ and $\pi^{\prime}\left(a^{\prime} \circ b^{\prime}\right)=\rho_{b^{\prime}}^{\prime}\left(\pi^{\prime}\left(a^{\prime}\right)\right) \oplus \pi^{\prime}\left(b^{\prime}\right)$, for all $a^{\prime}, b^{\prime} \in M(X, r)$ such that $\operatorname{deg}\left(a^{\prime}\right)+\operatorname{deg}\left(b^{\prime}\right)<\operatorname{deg}(a)+\operatorname{deg}(b)$.

Write $a=x \circ a^{\prime}$ and $b=b^{\prime} \circ y$ for some $x, y \in X$ and $a^{\prime}, b^{\prime} \in M(X, r)$. By the induction hypothesis we have

$$
\begin{aligned}
\pi(a \circ b) & =\pi\left(x \circ a^{\prime} \circ b\right) \\
& =x+\lambda_{x}^{\prime}\left(\pi\left(a^{\prime} \circ b\right)\right) \\
& =x+\lambda_{x}^{\prime}\left(\pi\left(a^{\prime}\right)+\lambda_{a^{\prime}}^{\prime}(\pi(b))\right) \\
& =x+\lambda_{x}^{\prime}\left(\pi\left(a^{\prime}\right)\right)+\lambda_{x}^{\prime}\left(\lambda_{a^{\prime}}^{\prime}(\pi(b))\right) \\
& =\pi\left(x \circ a^{\prime}\right)+\lambda_{x \circ a^{\prime}}^{\prime}(\pi(b)) \\
& =\pi(a)+\lambda_{a}^{\prime}(\pi(b))
\end{aligned}
$$

and

$$
\begin{aligned}
\pi^{\prime}(a \circ b) & =\pi^{\prime}\left(a \circ b^{\prime} \circ y\right) \\
& =\rho_{y}^{\prime}\left(\pi^{\prime}\left(a \circ b^{\prime}\right)\right) \oplus y \\
& =\rho_{y}^{\prime}\left(\rho_{b^{\prime}}^{\prime}\left(\pi^{\prime}(a)\right) \oplus \pi^{\prime}\left(b^{\prime}\right)\right) \oplus y \\
& =\rho_{y}^{\prime}\left(\rho_{b^{\prime}}^{\prime}\left(\pi^{\prime}(a)\right)\right) \oplus \rho_{y}^{\prime}\left(\pi^{\prime}\left(b^{\prime}\right)\right) \oplus y \\
& =\rho_{b^{\prime} \circ y}^{\prime}\left(\pi^{\prime}(a)\right) \oplus \pi^{\prime}\left(b^{\prime} \circ y\right) \\
& =\rho_{b}^{\prime}\left(\pi^{\prime}(a)\right) \oplus \pi^{\prime}(b) .
\end{aligned}
$$

Thus (19) and (20) follow by induction. It is clear that $\pi$ and $\pi^{\prime}$ are the unique 1-cocycles satisfying the hypothesis. Therefore the result follows.

A natural question is the following. 
Question 3.3 When are the 1-cocycles $\pi$ and $\pi^{\prime}$ bijective?

In general, these 1-cocycles are not bijective. We provide two examples. The first one is an example where $\pi$ is injective but not surjective, and the second one where $\pi$ and $\pi^{\prime}$ are neither injective nor surjective.

Example 3.4 Let $(X, r)$ be a set-theoretic solution of the YBE, where $X$ is set of cardinality greater than 1 and $r: X \times X \rightarrow X \times X$ is a map defined by $r(x, y)=(x, x)$, for all $x, y \in X$. The associated monoids are

$$
\begin{aligned}
& M(X, r)=\langle X| x \circ y=x \circ x, \text { for all } x, y \in X\rangle, \\
& A(X, r)=\langle X| x+x=x+x, \text { for all } x, y \in X\rangle, \\
& \left.A^{\prime}(X, r)=\langle X| x \oplus y=x \oplus x, \text { for all } x, y \in X\right\rangle .
\end{aligned}
$$

The 1-cocycle $\pi^{\prime}$ is bijective, but it is clear that the 1-cocycle $\pi$ is not. The latter is not surjective, for example the element $x+y$, where $x \neq y \in X$ is not in the image of $\pi$. Note that $\pi$ is still injective. Similarly, $(X, r)$ with $r: X \times X \rightarrow X \times X$ defined by $r(x, y)=(y, y)$ is an example of a set-theoretic solution of the

\begin{tabular}{|c|c|c|c|c|c|c|}
\hline$\wedge$ & 0 & 1 & 2 & V & 0 & 1 \\
\hline 0 & 0 & 0 & 0 & 0 & 0 & 1 \\
\hline 1 & 0 & 1 & 2 & 1 & 1 & 1 \\
\hline 2 & 0 & 1 & 2 & 2 & 2 & 2 \\
\hline
\end{tabular}
YBE where $\pi^{\prime}$ is injective but not surjective.

Example 3.5 Let $S=\{0,1,2\}$ and define the skew lattice $(S, \wedge, \vee)$ by

The skew lattice $(S, \wedge, \vee)$ is an example of a distributive and cancellative skew lattice that is not a co-strongly distributive skew lattice, see ([10, Example 4.4]). By [10, Theorem 5.7], $(S, \wedge, \vee)$ is a left distributive solution, i.e. $(S, r)$ is a set-theoretic solution of the YBE, where $r: S \times S \rightarrow S \times S$ is defined by $r(x, y)=(x \wedge y, y \vee x)$ for all $x, y \in S$. The associated monoids are

$$
\begin{aligned}
& M(X, r)=\langle 0,1,2 \mid 1 \circ 0=0 \circ 1,2 \circ 0=0 \circ 2,1 \circ 2=2 \circ 2,2 \circ 1=1 \circ 1\rangle, \\
& A(X, r)=\langle 0,1,2 \mid 1+0=0+0,2+0=0+0,1+2=2+2,2+1=1+1\rangle, \\
& A^{\prime}(X, r)=\langle 0,1,2 \mid 1 \oplus 0=1 \oplus 1,2 \oplus 0=2 \oplus 2\rangle .
\end{aligned}
$$

Both $\pi$ and $\pi^{\prime}$ are not injective, as $\pi(1 \circ 0)=1+0=0+0=\pi(0 \circ 0)$ and $\pi^{\prime}(1 \circ 0)=1 \oplus 0=1 \oplus 1=\pi^{\prime}(1 \circ 1)$, but $1 \circ 0 \neq 0 \circ 0$ and $1 \circ 0 \neq 1 \circ 1$ in $M(X, r)$. Both $\pi$ and $\pi^{\prime}$ are not surjective as $0+1$ (resp. $0 \oplus 1$ ) is not in the image of $\pi$ (resp. $\pi^{\prime}$ ).

Proposition 3.6 Let $(X, r)$ be a set-theoretic solution of the YBE. Write $r(x, y)=\left(\sigma_{x}(y), \gamma_{y}(x)\right)$. Let $\pi: M(X, r) \rightarrow A(X, r)$ and $\pi^{\prime}: M(X, r) \rightarrow A^{\prime}(X, r)$ be the 1-cocycles of Proposition 3.2. Then

(i) $\pi$ is surjective if and only if $\sigma_{x}$ is surjective for all $x \in X$,

(ii) $\pi^{\prime}$ is surjective if and only if $\gamma_{x}$ is surjective for all $x \in X$.

Proof. Suppose that $\sigma_{x}$ is surjective for all $x \in X$. First, we claim that $\sigma_{x}$ being surjective implies that $\lambda_{x}^{\prime}$ is surjective. Take $n$ an arbitrary positive integer. Let $x_{1}, \ldots, x_{n} \in X$ such that $x_{1}+\cdots+x_{n} \in A(X, r)$. As $\sigma_{x}$ is surjective, there exist $y_{1}, \ldots, y_{n} \in X$ such that $\sigma_{x}\left(y_{i}\right)=x_{i}$, for all $i \in\{1, \ldots, n\}$. Then, $\lambda_{x}^{\prime}\left(y_{1}+\cdots+y_{n}\right)=$ $\sigma_{x}\left(y_{1}\right)+\cdots+\sigma_{x}\left(y_{n}\right)=x_{1}+\cdots+x_{n}$, which proves that $\lambda_{x}^{\prime}$ is surjective.

Next, we prove that $\pi$ is surjective by induction on the length of the elements in $A(X, r)$. As $\pi(x)=x$ for all $x \in X, \pi$ is surjective on elements of length 1 . Assume now that for a fixed positive integer $n$ and for any $x_{1}, \ldots, x_{n} \in X$, there exist $y_{1}, \ldots, y_{n} \in X$ such that $\pi\left(y_{1} \circ \cdots \circ y_{n}\right)=x_{1}+\cdots+x_{n}$. Take $x_{1}, \ldots, x_{n+1} \in X$. Since $\lambda_{x_{1}}^{\prime}$ is surjective, there exists $z_{2}, \ldots, z_{n+1} \in X$ such that $\lambda_{x_{1}}^{\prime}\left(z_{2}+\cdots+z_{n+1}\right)=x_{2}+\cdots+x_{n+1}$. Using 
the induction hypotheses, there exists $y_{2}, \ldots, y_{n+1} \in X$ such that $\pi\left(y_{2} \circ \cdots \circ y_{n+1}\right)=z_{2}+\cdots+z_{n+1}$. Thus, we obtain

$$
\begin{aligned}
x_{1}+\cdots+x_{n+1} & =x_{1}+\lambda_{x_{1}}^{\prime}\left(z_{2}+\cdots+z_{n+1}\right) \\
& =x_{1}+\lambda_{x_{1}}^{\prime}\left(\pi\left(y_{2} \circ \cdots \circ y_{n+1}\right)\right) \\
& =\pi\left(x_{1} \circ y_{2} \circ \cdots \circ y_{n+1}\right),
\end{aligned}
$$

and $\pi$ is surjective.

Suppose now that $\pi$ is surjective. Let $x, y \in X$ and consider $x+y \in A(X, r)$. Since $\pi$ is surjective (and it preserves the degree), there exist $z, t \in X$ such that $\pi(z \circ t)=x+y$. Thus $z+\sigma_{z}(t)=x+y$ in $A(X, r)$. By the defining relations of $A(X, r)$, this equality implies that there exists $y^{\prime} \in X$ such that $\sigma_{x}\left(y^{\prime}\right)=y$. Therefore $\sigma_{x}$ is surjective for all $x \in X$.

The proof for $\pi^{\prime}$ is similar.

Proposition 3.7 Let $(X, r)$ be a set-theoretic solution of the YBE. Write $r(x, y)=\left(\sigma_{x}(y), \gamma_{y}(x)\right)$. Let $\pi: M(X, r) \rightarrow A(X, r)$ and $\pi^{\prime}: M(X, r) \rightarrow A^{\prime}(X, r)$ be the 1-cocycles of Proposition 3.2.

(i) If $\sigma_{x}$ is injective for all $x \in X$, then $\pi$ is injective.

(ii) If $\gamma_{x}$ is injective for all $x \in X$, then $\pi^{\prime}$ is injective.

Proof. We shall prove $(i)$. The proof of $(i i)$ is similar. Let $\operatorname{FM}(X)$ be the (multiplicative) free monoid on $X$. Suppose that $\sigma_{x}$ is injective for all $x \in X$. Since $\pi(x)=x$ for all $x \in X$, the restriction of $\pi$ to elements of degree one in $M(X, r)$ is injective. Let $n$ be an integer greater than 1 . Let $x_{1}, \ldots, x_{n}, y_{1}, \ldots, y_{n} \in X$ be elements such that $\pi\left(x_{1} \circ \cdots \circ x_{n}\right)=\pi\left(y_{1} \circ \cdots \circ y_{n}\right)$. Thus, in $A(X, r)$, we have that

$$
x_{1}+\sigma_{x_{1}}\left(x_{2}\right)+\cdots+\sigma_{x_{1}} \cdots \sigma_{x_{n-1}}\left(x_{n}\right)=y_{1}+\sigma_{y_{1}}\left(y_{2}\right)+\cdots+\sigma_{y_{1}} \cdots \sigma_{y_{n-1}}\left(y_{n}\right) \text {. }
$$

Let $w_{1}, w_{2} \in \operatorname{FM}(X)$ be two elements of degree $n$. Suppose that $w_{1}=z_{1} \cdots z_{n}$ and $w_{2}=t_{1} \cdots t_{n}$, for some $z_{i}, t_{i} \in X$. We say that $w_{1} \sim w_{2}$ if there exist $1 \leq i \leq n-1$ and $z \in X$ such that $z_{j}=t_{j}$, for all $j \in\{1,2, \ldots, n\} \backslash\{i, i+1\}$ and either $z_{i+1}=\sigma_{z_{i}}(z)=t_{i}$ and $t_{i+1}=\sigma_{t_{i}} \gamma_{z}\left(z_{i}\right)$, or $t_{i+1}=\sigma_{t_{i}}(z)=z_{i}$ and $z_{i+1}=\sigma_{z_{i}} \gamma_{z}\left(t_{i}\right)$. Note that $z_{1}+\cdots+z_{n}=t_{1}+\cdots+t_{n}$ in $A(X, r)$ if and only if there exist $w_{1}^{\prime}, \ldots, w_{m}^{\prime} \in \mathrm{FM}(X)$ of degree $n$ such that

$$
w_{1}=w_{1}^{\prime} \sim w_{2}^{\prime} \sim \cdots \sim w_{m}^{\prime}=w_{2}
$$

Hence to prove that $x_{1} \circ \cdots \circ x_{n}=y_{1} \circ \cdots \circ y_{n}$, we may assume that there exist $1 \leq i \leq n-1$ and $z \in X$ such that $\sigma_{x_{1}} \cdots \sigma_{x_{j-1}}\left(x_{j}\right)=\sigma_{y_{1}} \cdots \sigma_{y_{j-1}}\left(y_{j}\right)$, for all $j \in\{1,2, \ldots, n\} \backslash\{i, i+1\}$, and also $\sigma_{x_{1}} \cdots \sigma_{x_{i}}\left(x_{i+1}\right)=$ $\sigma_{\sigma_{x_{1}} \cdots \sigma_{x_{i-1}}\left(x_{i}\right)}(z)=\sigma_{y_{1}} \cdots \sigma_{y_{i-1}}\left(y_{i}\right)$ as well as $\sigma_{y_{1}} \cdots \sigma_{y_{i}}\left(y_{i+1}\right)=\sigma_{\sigma_{y_{1}} \cdots \sigma_{y_{i-1}}\left(y_{i}\right)} \gamma_{z}\left(\sigma_{x_{1}} \cdots \sigma_{x_{i-1}}\left(x_{i}\right)\right)$.

Since $\sigma_{x_{1}} \cdots \sigma_{x_{j-1}}\left(x_{j}\right)=\sigma_{y_{1}} \cdots \sigma_{y_{j-1}}\left(y_{j}\right)$, for all $j \in\{1,2, \ldots, n\} \backslash\{i, i+1\}$, and $\sigma_{x}$ is injective for all $x \in X$, we have that $x_{j}=y_{j}$, for all $j \in\{1, \ldots, i-1\}$. Hence, since $\sigma_{x_{1}} \cdots \sigma_{x_{i}}\left(x_{i+1}\right)=\sigma_{y_{1}} \cdots \sigma_{y_{i-1}}\left(y_{i}\right)$, and $\sigma_{x}$ is injective for all $x \in X$, we have that $y_{i}=\sigma_{x_{i}}\left(x_{i+1}\right)$. Now we have that

$$
\begin{aligned}
\sigma_{\sigma_{x_{1}} \cdots \sigma_{x_{i-1}}\left(x_{i}\right)}(z) & =\sigma_{x_{1}} \cdots \sigma_{x_{i}}\left(x_{i+1}\right) \\
& =\lambda_{x_{1} \circ \cdots \circ x_{i-1}} \lambda_{x_{i}}\left(x_{i+1}\right) \\
& =\lambda_{\lambda_{x_{1} \circ \cdots \circ x_{i-1}}\left(x_{i}\right)} \lambda_{\rho_{x_{i}}\left(x_{1} \circ \cdots \circ x_{i-1}\right)}\left(x_{i+1}\right) \\
& =\sigma_{\sigma_{x_{1}} \cdots \sigma_{x_{i-1}}\left(x_{i}\right)} \lambda_{\rho_{x_{i}}\left(x_{1} \circ \cdots \circ x_{i-1}\right)}\left(x_{i+1}\right)
\end{aligned}
$$

where the third equality follows by Theorem 2.1 .

Hence, since $\sigma_{x}$ is injective for all $x \in X$, we have that

$$
z=\lambda_{\rho_{x_{i}}\left(x_{1} \circ \cdots \circ x_{i-1}\right)}\left(x_{i+1}\right) .
$$


By Theorem 2.1

$$
\begin{aligned}
\sigma_{y_{1}} & \cdots \sigma_{y_{i}}\left(y_{i+1}\right) \\
& =\sigma_{\sigma_{y_{1}} \cdots \sigma_{y_{i-1}}\left(y_{i}\right)} \gamma_{z}\left(\sigma_{x_{1}} \cdots \sigma_{x_{i-1}}\left(x_{i}\right)\right) \\
& =\sigma_{\sigma_{x_{1}} \cdots \sigma_{x_{i-1}}}\left(\sigma_{x_{i}}\left(x_{i+1}\right)\right) \\
& =\gamma_{z}\left(\sigma_{x_{1}} \cdots \sigma_{x_{i-1}}\left(x_{i}\right)\right) \\
& =\lambda_{\lambda_{x_{1}} \circ \cdots \circ x_{i-1}}\left(\lambda_{x_{i}}\left(x_{i+1}\right)\right) \rho_{\lambda_{\rho_{x_{i}}\left(x_{1} \circ \cdots \circ x_{i-1}\right)}\left(x_{i+1}\right)}\left(\lambda_{x_{1} \circ \cdots \circ x_{i-1}}\left(x_{i}\right)\right) \\
& =\lambda_{\lambda_{x_{1}} \circ \cdots \circ x_{i-1}}\left(\lambda_{x_{i}}\left(x_{i+1}\right)\right) \\
& \lambda_{\rho_{\lambda_{x_{i}}\left(x_{i+1}\right)}\left(x_{1} \circ \cdots \circ x_{i-1}\right)}\left(\rho_{x_{i+1}}\left(x_{i}\right)\right) \\
& =\lambda_{x_{1} \circ \cdots \circ x_{i-1}} \lambda_{\lambda_{x_{i}}\left(x_{i+1}\right)}\left(\rho_{x_{i+1}}\left(x_{i}\right)\right) \\
& =\sigma_{y_{1} \circ \cdots \circ y_{i-1}} \lambda_{y_{i}}\left(\rho_{x_{i+1}}\left(x_{i}\right)\right) \\
&
\end{aligned}
$$

Since $\sigma_{x}$ is injective for all $x \in X$, we have that $y_{i+1}=\gamma_{x_{i+1}}\left(x_{i}\right)$. Thus,

$$
y_{i} \circ y_{i+1}=\sigma_{x_{i}}\left(x_{i+1}\right) \circ \gamma_{x_{i+1}}\left(x_{i}\right)=x_{i} \circ x_{i+1} \text {. }
$$

Since $\sigma_{x_{1}} \cdots \sigma_{x_{j-1}}\left(x_{j}\right)=\sigma_{y_{1}} \cdots \sigma_{y_{j-1}}\left(y_{j}\right)$, for all $j \in\{1,2, \ldots, n\} \backslash\{i, i+1\}$, and $\sigma_{x}$ is injective for all $x \in X$, we have that $x_{j}=y_{j}$, for all $j \in\{i+2, \ldots, n\}$. Hence $x_{1} \circ \cdots \circ x_{n}=y_{1} \circ \cdots \circ y_{n}$, and therefore, $\pi$ is injective.

Remark 3.8 Note that in the set-theoretic solution of the YBE of Example 3.4 . $\sigma_{x}(y)=x$ for all $x, y \in X$, so $\sigma_{x}$ is not injective. But, $\pi$ is injective. Similarly, $(X, r)$ with $r: X \times X \rightarrow X \times X$ defined by $r(x, y)=(y, y)$ is a set-theoretic solution of the YBE where $\pi^{\prime}$ is injective (see Example 3.4) but $\gamma_{y}(x)=y$ for all $x, y \in X$. So $\gamma_{y}$ is not injective.

If $\pi$ (resp. $\pi^{\prime}$ ) is injective, then it is clear that the map $f$ (resp. $f^{\prime}$ ), defined in Proposition 3.2 is an embedding. The latter was proved in [19] under the assumption that $(X, r)$ is a left non-degenerate solution. In this case $\pi$ is bijective and $M(X, r)$ is a regular submonoid of the semidirect $\operatorname{product} A(X, r) \rtimes \operatorname{gr}\left(\sigma_{x} \mid\right.$ $x \in X)$.

The following result answers Question 3.3 for finite solutions.

Corollary 3.9 [19] (Jespers, Kubat and Van Antwerpen) Let (X,r) be a set-theoretic solution of the YBE, $\lambda^{\prime}$ (resp. $\left.\rho^{\prime}\right)$ the left (resp. right) action as defined before, $\pi$ (resp. $\pi^{\prime}$ ) the unique 1-cocycle with respect to $\lambda^{\prime}\left(\right.$ resp. $\left.\rho^{\prime}\right)$. Then, $\pi$ (resp. $\left.\pi^{\prime}\right)$ is bijective if $(X, r)$ is left non-degenerate (resp. right non-degenerate). The converse holds if $X$ is finite.

Proof. Assume first that $(X, r)$ is a left non-degenerate set-theoretic solution of the YBE. Then, by Propositions 3.6 and 3.7 $\pi$ is bijective. Similarly, one can prove that $(X, r)$ being a right non-degenerate solution implies that $\pi^{\prime}$ is bijective.

Assume now that $\pi: M(X, r) \rightarrow A(X, r)$ is bijective and $X$ is finite. By Proposition 3.6, $\sigma_{x}$ is surjective for all $x \in X$. Since $X$ is finite, $\sigma_{x}$ is bijective for all $x \in X$, that is $(X, r)$ is left non-degenerate.

The next example shows the difficulty of Question 3.3 for infinite solutions.

Example 3.10 Consider the set $\mathbb{N}$ of the non-negative integers. Let $r: \mathbb{N} \times \mathbb{N} \longrightarrow \mathbb{N} \times \mathbb{N}$ be the map defined by $r(x, y)=(\xi(y), \xi(x))$, for all $x, y \in \mathbb{N}$, where $\xi(x)=\max \{0, x-1\}$, for all $x \in \mathbb{N}$. Then $(\mathbb{N}, r)$ is a set-theoretic solution of the YBE, such that the associated 1-cocycles $\pi$ and $\pi^{\prime}$ are bijective, but, for every $x \in \mathbb{N} \sigma_{x}=\gamma_{x}=\xi$ is not injective, because $\xi(0)=\xi(1)$.

Proof. It is easy to check that $(\mathbb{N}, r)$ is a set-theoretic solution of the YBE. Note that, for every $x \in \mathbb{N}$, $\xi^{x}(x)=0$. Hence

$$
M(\mathbb{N}, r)=\langle\mathbb{N} \mid x \circ y=0 \circ 0\rangle,
$$




$$
A(\mathbb{N}, r)=\langle\mathbb{N} \mid x+y=0+0\rangle
$$

and

$$
A^{\prime}(\mathbb{N}, r)=\langle\mathbb{N} \mid x \oplus y=0 \oplus 0\rangle .
$$

Therefore, for every integer $n>1$, the monoids $M(\mathbb{N}, r), A(\mathbb{N}, r)$ and $A^{\prime}(\mathbb{N}, r)$ have only one element of degree $n$. Since $\pi$ and $\pi^{\prime}$ preserve the degree and $\pi(x)=x$ and $\pi^{\prime}(x)=x$, for all $x \in \mathbb{N}$, we have that $\pi$ and $\pi^{\prime}$ are bijective. Thus the result follows.

\section{Non-degenerate irretractable solutions}

In [26. Theorem 2] (and independently in [20, Corollary 2.3]), it is proven that any finite involutive left non-degenerate set-theoretic solution of the YBE also is right non-degenerate. In the infinite case, the latter is no longer true. The following example from [26] shows this.

Example 4.1 Let $\mathrm{X}$ be the set of the integers, and define $r: X^{2} \rightarrow X^{2}$ by

$$
r(x, y)=\left(\lambda_{x}(y), \lambda_{\lambda_{x}(y)}^{-1}(x)\right)
$$

where $\lambda_{x}(y)=y+\min (x, 0)$, for all $x, y \in X$. Note that $\lambda_{x}$ is bijective and $\lambda_{x}^{-1}(y)=y-\min (x, 0)$. It is easy to check that $(X, r)$ is an involutive solution. Note that it is not right non-degenerate. In fact, if $a<0$, we have that

$$
\rho_{a}(b)=\lambda_{\lambda_{b}(a)}^{-1}(b)=b-\min (a+\min (b, 0), 0)=b-(a+b)=-a,
$$

for all $b<0$. Hence $\rho_{a}$ is not bijective if $a<0$.

It is unclear whether the above holds for aribtrary bijective solutions. Hence the following question is pertinent.

Question 4.2 Is any finite bijective left non-degenerate set-theoretic solution of the YBE right non-degenerate?

A natural question is the converse:

Question 4.3 Are non-degenerate solutions of the YBE always bijective?

We will give a positive answer to this question in case the solution $(X, r)$ is irretractable, i.e. $\sigma_{x}=\sigma_{y}$ implies $x=y$, for all $x, y \in X$. Note that Example 4.1 is a retractable involutive solution. To our knowledge it is unknown whether there exist infinite involutive irretractable solutions that are left but not right nondegenerate. Note that irretractability has been defined with respect to to the maps $\sigma_{x}$. One could equally well define retractabilty with respect to the maps $\gamma_{x}$. However, this makes no difference since any solution $r(x, y)=\left(\sigma_{x}(y), \gamma_{y}(x)\right)$ has a dual solution $r^{\prime}(y, x)=\left(\gamma_{y}(x), \sigma_{x}(y)\right)$. Clearly $r$ is (bijective) non-degenerate if and only if $r^{\prime}$ is (bijective) non-degenerate.

To prove the result we will make use of the following result of Rump [29, Proposition 1]: Let $X$ be a non-empty set and let $r: X \times X \rightarrow X \times X$ be a map, with $r(x, y)=\left(\sigma_{x}(y), \gamma_{y}(x)\right)$, such that $\gamma_{y}: X \rightarrow X$ is bijective for all $y \in X$. Then $(X, r)$ is a solution of the YBE if and only if the following conditions hold for all $x, y, z \in X$ :

(R1) $(x \cdot y) \cdot(x \cdot z)=(y: x) \cdot(y \cdot z)$,

(R2) $(x: y):(x: z)=(y \cdot x):(y: z)$,

(R3) $(x \cdot y):(x \cdot z)=(y: x) \cdot(y: z)$, 
where $x \cdot y=\gamma_{x}^{-1}(y), x: y=\sigma_{\gamma_{y}^{-1}(x)}(y)$. Furthermore, $r$ is a bijective solution if the map $X \rightarrow X$ defined by $z \mapsto x: z$ is bijective. The use of this result has been proposed by the referee to avoid the arboresque sub- and superscripts in the original proof.

We also will make use of a lemma that was proved by Lebed and Vendramin in [24] for finite nondegenerate bijective solutions.

Lemma 4.4 Let $(X, r)$ be a non-degenerate set-theoretic solution of the YBE. Let $h: X \rightarrow X$ be the map defined by $h(x)=\sigma_{x}^{-1}(x)$, for all $x \in X$. If $(X, r)$ is irretractable, then $h$ is bijective and $h^{-1}(x)=\gamma_{x}^{-1}(x)$, for all $x \in X$.

Proof. As commented above, we may assume that $(X, r)$ is a non-degenerate set-theoretic solution of the YBE such that $\gamma_{x}=\gamma_{y}$ implies that $x=y$. Thus conditions (R1),(R2),(R3) hold. Then, by (R1),

$$
\gamma_{x \cdot x}^{-1}(x \cdot z)=\gamma_{x: x}^{-1}(x \cdot z)
$$

for all $x, z \in X$. Hence,

$$
x \cdot x=x: x .
$$

Now $x: x=\sigma_{x \cdot x}(x)$ and thus $\sigma_{x \cdot x}^{-1}(x \cdot x)=x$. This shows that the map $x \mapsto x \cdot x=\gamma_{x}^{-1}(x)$ is injective. For $x, y \in X$, put

$$
\sigma_{x}^{-1}(y)=x * y
$$

For $y=x * x$, we have $x=\sigma_{x}(y)=\sigma_{\gamma_{y}^{-1}\left(\gamma_{y}(x)\right)}(y)=\gamma_{y}(x): y$. Hence by $(\mathrm{R} 1)$

$$
x \cdot\left(\gamma_{y}(x) \cdot z\right)=\left(\gamma_{y}(x): y\right) \cdot\left(\gamma_{y}(x) \cdot z\right)=\left(y \cdot \gamma_{y}(x)\right) \cdot(y \cdot z)=x \cdot(y \cdot z)
$$

Therefore $\gamma_{y}(x) \cdot z=y \cdot z$, which yields $\gamma_{y}(x)=y$. Hence

$$
x=y \cdot y=(x * x) \cdot(x * x),
$$

which shows that the map $x \mapsto x \cdot x=\gamma_{x}^{-1}(x)$ is bijective. Furthermore the inverse of this map is the map $x \mapsto x * x=\sigma_{x}^{-1}(x)=h(x)$.

Theorem 4.5 Let $(X, r)$ be an irretractable non-degenerate set-theoretic solution of the YBE. Then $r$ is bijective.

Proof. Again we may assume that $(X, r)$ is a non-degenerate set-theoretic solution of the YBE such that $\gamma_{x}=\gamma_{y}$ implies that $x=y$. From (R3) we get that

$$
x:(z \cdot z)=\left(z \cdot \gamma_{z}(x)\right):(z \cdot z)=\left(\gamma_{z}(x): z\right) \cdot\left(\gamma_{z}(x): z\right)=\sigma_{x}(z) \cdot \sigma_{x}(z) .
$$

From Lemma 4.4 we then get that (see equation (21) )

$$
x: z=\sigma_{x}(z * z) \cdot \sigma_{x}(z * z)
$$

Since $x * x=\sigma_{x}^{-1}(x)=h(x)$, we get from Lemma 4.4 that the map $z \mapsto x: z=\sigma_{\gamma_{z}^{-1}(x)}(z)$ is bijective. Hence, by Rump's earlier mentioned result, $r$ is bijective.

Note that if $(X, r)$ is an irretractable non-degenerate solution then for every $x \in X$ there is a unique $y \in X$ such that $r(x, y)=(x, y)$ and there is a unique $z \in X$ such that $r(z, x)=(z, x)$. Because $(X, r)$ is left non-degenerate, to prove the former, it is sufficient to show that $\sigma_{x}(y)=x$ implies $\gamma_{y}(x)=y$. Now, because $(X, r)$ is a solution we obtain from (11) that $\sigma_{x} \sigma_{y}=\sigma_{\sigma_{x}(y)} \sigma_{\gamma_{y}(x)}=\sigma_{x} \sigma_{\gamma_{y}(x)}$ and thus $\sigma_{y}=\sigma_{\gamma_{y}(x)}$. The irretractble assumption yields that $y=\gamma_{y}(x)$, as claimed. Similarly one proves the other claim. Hence the there are at least $\left(\begin{array}{c}|X| \\ 2\end{array}\right)$ defining relations for the structure monoid. Furthermore, there are precisely 
$\left(\begin{array}{c}|X| \\ 2\end{array}\right)$ defining relations if $r$ also is involutive and thus, in this case, $M(X, r)$ is a monoid with a presentation of the type $\left\langle x_{1}, \ldots, x_{n} \mid R\right\rangle$, where $R$ is a set consisting of $\left(\begin{array}{l}n \\ 2\end{array}\right)$ relations of the type $x_{i} x_{j}=x_{k} x_{l}$ with $\left(x_{i}, x_{j}\right) \neq\left(x_{k}, x_{l}\right)$ and every word $x_{i} x_{j}$ appears in at most one relation. Note that such a presentation has associated a map $r: X \times X \rightarrow X \times X$, where $X=\left\{x_{1}, \ldots x_{n}\right\}, r^{2}=\operatorname{id}_{X^{2}}$ and $r\left(x_{i}, x_{j}\right)=\left(x_{k}, x_{l}\right)$ if and only if either $x_{i} x_{j}=x_{k} x_{l}$ is one of the relations in $R$ or $x_{i} x_{j}$ does not appear in any relation in $R$ and $\left(x_{k}, x_{l}\right)=\left(x_{i}, x_{j}\right)$ in this case. Monoids with this type of presentation and their algebras have a rich algebraic structure, when $r$ is non-degenerate, even if $(X, r)$ is not a solution of the YBE. Such monoids are said to be of quadratic type, and if $x_{i} x_{i}$ does not appear in any defining relation, then they are said to be of skew type. We refer the reader to [6, 15, 22, 23. In [22] it has been shown that for such a monoid $r$ is a non-degenerate solution of the YBE if and only if the monoid is cancellative and $r$ is non-degenerate and satisfies the cyclic condition, i.e. if for every $x_{1}, y \in X$ there exist $x_{2}, y_{1}, y_{2}, z_{1}, z_{2} \in X$ such that $x_{1} y=y_{1} z_{1}$ and $x_{2} y_{1}=y_{2} z_{2}$ with $r\left(x_{2}, x_{1}\right)=\left(x_{2}, x_{1}\right)$ and $r\left(z_{2}, z_{1}\right)=\left(z_{2}, z_{1}\right)$. The latter monoids were first investigated by Gateva-Ivanova and Van den Bergh in [17.

\section{$5 \quad$ The structure left semi-truss}

Braces and skew braces were introduced to deal with bijective non-degenerate solutions $(X, r)$ of the YBE. In order to translate such solutions to associative structures the structure group $G(X, r)$ and the structure monoid $M(X, r)$ were introduced. The group $G(X, r)$ turns out to be a skew brace, however a structure monoid does not fit in this context. Recently, Brzeziński introduced the algebraic notion of a semi-truss which is built on two semigroup structures on a given set. We show that structure monoids of left non-degenerate solutions of the YBE fit in this context: they turn out to be left semi-trusses with additive structure that is close to being a normal monoid. We then show that also the least left cancellative epimorphic image of $M(X, r)$ inherits a left non-degenerate solution of the YBE that restricts to the original solution $r$ for some interesting class, in particular if $(X, r)$ is irretractable.

We first recall the definition of a left semi-truss.

Definition 5.1 [4] A left semi-truss is a quadruple $(A,+, \circ, \phi)$ such that $(A,+)$ and $(A, \circ)$ are non-empty semigroups and $\phi: A \times A \longrightarrow A$ is a function such that

$$
a \circ(b+c)=(a \circ b)+\phi(a, c),
$$

for all $a, b, c \in A$.

Example 5.2 Let $(X, r)$ be a left non-degenerate set-theoretic solution of the YBE (not necessarily bijective). As stated in Section 3, and with the same notation, the map $r^{\prime}(x, y)=\left(y, \sigma_{y} \gamma_{\sigma_{x}^{-1}(y)}(x)\right)$ defines the left derived solution on $X$. Let $M=M(X, r)$ and $M^{\prime}=A(X, r)=M\left(X, r^{\prime}\right)$ be the structure monoids of the solutions $(X, r)$ and $\left(X, r^{\prime}\right)$ respectively. From Corollary 3.9 and Proposition 3.1 we obtain a left action $\lambda^{\prime}:(M, \circ) \longrightarrow \operatorname{Aut}\left(M^{\prime},+\right)$ and a bijective 1-cocycle $\pi: M \longrightarrow M^{\prime}$ with respect to $\lambda^{\prime}$ satisfying $\lambda^{\prime}(x)(y)=\sigma_{x}(y)$ and $\pi(x)=x$, for all $x, y \in X$. We identify $M$ and $M^{\prime}$ via $\pi$, that is $a=\pi(a)$ for all $a \in M$. With this identification, we obtain the operation + on $M$, and $a \circ b=a+\lambda_{a}^{\prime}(b)$, for all $a, b \in M$. Put $\phi(a, b)=\lambda_{a}^{\prime}(b)$, for all $a, b \in M$. Then

$$
a \circ(b+c)=a+\lambda_{a}^{\prime}(b+c)=a+\lambda_{a}^{\prime}(b)+\lambda_{a}^{\prime}(c)=(a \circ b)+\phi(a, c) .
$$

Furthermore $M+a \subseteq a+M$, for all $a \in M$. Hence $(M,+, \circ, \phi)$ is a left semi-truss. Note that if, furthermore, $r$ is bijective then it easily can be verified that $\left(X, r^{\prime}\right)$ is a right non-degenerate solution and thus $M+a=a+M$ for all $a \in M$; that is $(M,+)$ consists of normal elements. As shown in [19, this property is fundamental in the study of the associated structure algebra $K M(X, r)$.

In the remainder of this section we show that if $(M,+, \circ, \phi)$ is a left semi-truss such that for every $a, b \in M$ there exists a unique $c(a, b) \in M$ such that $a+b=b+c(a, b)$, then there exists a set-theoretical solution of the YBE on $M$, say $\left(M, r^{\prime}\right)$. In case that $M=M(X, r) / \eta$, the least cancellative epimorphic image of $M(X, r)$ it follows that $r^{\prime}$ is the (unique) extension of $r$ to $M$. 
Lemma 5.3 Let $(A,+)$ be a non-empty semigroup such that for each $(a, b) \in A \times A$ there exists a unique $c(a, b) \in A$ such that

$$
a+b=b+c(a, b)
$$

Then $\left(A, r^{\prime}\right)$, where

$$
r^{\prime}(a, b)=(b, c(a, b)),
$$

for all $a, b \in A$, is a set-theoretic solution of the $Y B E$.

Proof. Let $(a, b, d) \in A^{3}$. We have

$$
\begin{aligned}
a+b+d & =b+c(a, b)+d \\
& =b+d+c(c(a, b), d)
\end{aligned}
$$

and also

$$
\begin{aligned}
a+b+d & =a+d+c(b, d) \\
& =d+c(a, d)+c(b, d) \\
& =d+c(b, d)+c(c(a, d), c(b, d)) \\
& =b+d+c(c(a, d), c(b, d)) .
\end{aligned}
$$

Hence, by the uniqueness assumption,

$$
c(a, b+d)=c(c(a, b), d)=c(c(a, d), c(b, d)) .
$$

Now we have

$$
r_{1}^{\prime} r_{2}^{\prime} r_{1}^{\prime}(a, b, d)=r_{1}^{\prime} r_{2}^{\prime}(b, c(a, b), d)=r_{1}^{\prime}(b, d, c(c(a, b), d))=(d, c(b, d), c(c(a, b), d))
$$

and

$$
\begin{aligned}
r_{2}^{\prime} r_{1}^{\prime} r_{2}^{\prime}(a, b, d) & =r_{2}^{\prime} r_{1}^{\prime}(a, d, c(b, d))=r_{2}^{\prime}(d, c(a, d), c(b, d)) \\
& =(d, c(b, d), c(c(a, d), c(b, d))) .
\end{aligned}
$$

Therefore, by (22) $r_{1}^{\prime} r_{2}^{\prime} r_{1}^{\prime}=r_{2}^{\prime} r_{1}^{\prime} r_{2}^{\prime}$, and the result follows.

Proposition 5.4 Let $(A,+)$ and $(A, \circ)$ be non-empty semigroups. Let $\lambda:(A, \circ) \rightarrow \operatorname{Aut}(A,+)$ be a homomorphism such that $a \circ b=a+\lambda_{a}(b)$, for all $a, b \in A$, where $\lambda(a)=\lambda_{a}$. In particular, $(A,+, \circ, \phi)$ is a left semi-truss with $\phi(a, b)=\lambda_{a}(b)$, for all $a, b \in A$. Suppose that for each $(a, b) \in A \times A$ there exists a unique $c(a, b) \in A$ such that

$$
a+b=b+c(a, b)
$$

Then $(A, r)$, where

$$
r(a, b)=\left(\lambda_{a}(b), \lambda_{\lambda_{a}(b)}^{-1}\left(c\left(a, \lambda_{a}(b)\right)\right)\right)
$$

for all $a, b \in A$, is a left non-degenerate set-theoretic solution of the YBE.

Proof. Let $J: A^{3} \longrightarrow A^{3}$ be the map defined by $J(a, b, d)=\left(a, \lambda_{a}(b), \lambda_{a} \lambda_{b}(d)\right)$. Clearly $J$ is bijective and $J^{-1}(a, b, d)=\left(a, \lambda_{a}^{-1}(b), \lambda_{\lambda_{a}^{-1}(b)}^{-1} \lambda_{a}^{-1}(d)\right)$, for all $a, b, d \in A$. We have

$$
\begin{aligned}
J^{-1} r_{1}^{\prime} J(a, b, d) & =J^{-1} r_{1}^{\prime}\left(a, \lambda_{a}(b), \lambda_{a} \lambda_{b}(d)\right) \\
& =J^{-1}\left(\lambda_{a}(b), c\left(a, \lambda_{a}(b)\right), \lambda_{a} \lambda_{b}(d)\right) \\
& =\left(\lambda_{a}(b), \lambda_{\lambda_{a}(b)}^{-1}\left(c\left(a, \lambda_{a}(b)\right)\right), \lambda_{\lambda_{\lambda_{a}(b)}^{-1}\left(c\left(a, \lambda_{a}(b)\right)\right)}^{-1} \lambda_{\lambda_{a}(b)}^{-1} \lambda_{a} \lambda_{b}(d)\right),
\end{aligned}
$$


where $r^{\prime}$ is defined as in Lemma 5.3 Since $a \circ b=a+\lambda_{a}(b)=\lambda_{a}(b)+c\left(a, \lambda_{a}(b)\right)=\lambda_{a}(b) \circ \lambda_{\lambda_{a}(b)}^{-1}\left(c\left(a, \lambda_{a}(b)\right)\right)$ it follows that $J^{-1} r_{1}^{\prime} J=r_{1}$. Similarly

$$
\begin{aligned}
J^{-1} r_{2}^{\prime} J(a, b, d) & =J^{-1} r_{2}^{\prime}\left(a, \lambda_{a}(b), \lambda_{a} \lambda_{b}(d)\right) \\
& =J^{-1}\left(a, \lambda_{a} \lambda_{b}(d), c\left(\lambda_{a}(b), \lambda_{a} \lambda_{b}(d)\right)\right) \\
& =\left(a, \lambda_{b}(d), \lambda_{\lambda_{b}(d)}^{-1} \lambda_{a}^{-1}\left(c\left(\lambda_{a}(b), \lambda_{a} \lambda_{b}(d)\right)\right)\right)
\end{aligned}
$$

Note that

$$
\lambda_{a}^{-1}(d)+\lambda_{a}^{-1}(c(b, d))=\lambda_{a}^{-1}(d+c(b, d))=\lambda_{a}^{-1}(b+d)=\lambda_{a}^{-1}(b)+\lambda_{a}^{-1}(d),
$$

for all $a, b, d \in A$. Hence, by the uniqueness assumption, $\lambda_{a}^{-1}(c(b, d))=c\left(\lambda_{a}^{-1}(b), \lambda_{a}^{-1}(d)\right)$. Since each $\lambda_{a}$ is bijective it follows that

$$
\begin{aligned}
J^{-1} r_{2}^{\prime} J(a, b, d) & =\left(a, \lambda_{b}(d), \lambda_{\lambda_{b}(d)}^{-1} \lambda_{a}^{-1}\left(c\left(\lambda_{a}(b), \lambda_{a} \lambda_{b}(d)\right)\right)\right) \\
& =\left(a, \lambda_{b}(d), \lambda_{\lambda_{b}(d)}^{-1}\left(c\left(b, \lambda_{b}(d)\right)\right)\right) .
\end{aligned}
$$

Thus $J^{-1} r_{2}^{\prime} J=r_{2}$. By Lemma $5.3\left(A, r^{\prime}\right)$ is a set-theoretic solution of the YBE. Therefore also $(A, r)$ is a set-theoretic solution of the YBE, and the result follows.

Let $(X, r)$ be a left non-degenerate set-theoretic solution of the YBE. We will write $r(x, y)=\left(\sigma_{x}(y), \gamma_{y}(x)\right)$, for all $x, y \in X$. Thus the $\sigma_{x}$ are bijective maps. The derived solution of $(X, r)$ is $\left(X, r^{\prime}\right)$, where

$$
r^{\prime}(x, y)=\left(y, \sigma_{y}\left(\gamma_{\sigma_{x}^{-1}(y)}(x)\right)\right),
$$

for all $x, y \in X$. We will use the notation of Example 5.2. Thus we have $M=M(X, r)$ and the left semi-truss $(M,+, \circ, \phi)$, where $\phi(a, b)=\lambda_{a}^{\prime}(b)$, for all $a, b \in M$. Recall that $\lambda^{\prime}:(M, \circ) \longrightarrow \operatorname{Aut}(M,+)$ is an homomorphism, that is, an action of $(M, \circ)$ on $(M,+)$, and id: $M \longrightarrow M$ is a bijective 1-cocycle with respect to $\lambda^{\prime}$ (because $\left.a \circ b=a+\lambda_{a}^{\prime}(b)\right)$.

Let $\eta$ be the left cancellative congruence on $(M,+)$, that is, $\eta$ is the smallest congruence such that $\bar{M}=(M,+) / \eta$ is a left cancellative monoid.

We shall see a description of the elements in $\eta$. Let

$$
\eta_{0}=\left\{(a, b) \in M^{2} \mid \exists c \in M \text { such that } c+a=c+b\right\} .
$$

Note that $\eta_{0}$ is a reflexive and symmetric binary relation on $M$. Let $\eta_{1}$ be its transitive closure, that is

$$
\eta_{1}=\left\{(a, b) \in M^{2} \mid \exists a_{1}, \ldots, a_{n} \in M \text { such that }\left(a, a_{1}\right),\left(a_{1}, a_{2}\right), \ldots,\left(a_{n}, b\right) \in \eta_{0}\right\} .
$$

Thus $\eta_{1}$ is an equivalence relation on $M$. Let

$$
\begin{aligned}
\eta_{2}= & \left\{(c+a, c+b) \in M^{2} \mid c \in M \text { such that }(a, b) \in \eta_{1}\right\} \\
& \cup\left\{(a, b) \in M^{2} \mid \exists c \in M \text { such that }(c+a, c+b) \in \eta_{1}\right\},
\end{aligned}
$$

and for every $m \geq 1$ we define

$$
\eta_{2 m+1}=\left\{(a, b) \in M^{2} \mid \exists a_{1}, \ldots, a_{n} \in M \text { such that }\left(a, a_{1}\right),\left(a_{1}, a_{2}\right), \ldots,\left(a_{n}, b\right) \in \eta_{2 m}\right\}
$$

and

$$
\begin{aligned}
\eta_{2 m+2}= & \left\{(c+a, c+b) \in M^{2} \mid c \in M \text { such that }(a, b) \in \eta_{2 m+1}\right\} \\
& \cup\left\{(a, b) \in M^{2} \mid \exists c \in M \text { such that }(c+a, c+b) \in \eta_{2 m+1}\right\},
\end{aligned}
$$

Note that $\eta_{n} \subseteq \eta_{n+1} \subseteq \eta$ for all $n \geq 0$. Let $\eta^{\prime}=\cup_{n=0}^{\infty} \eta_{n}$. 
Lemma 5.5 With the above notation we have that $\eta^{\prime}=\eta$ and $\lambda_{a}^{\prime}=\lambda_{b}^{\prime}$, for all $(a, b) \in \eta$. Furthermore, for all $z \in M$,

$$
\eta=\left\{\left(\lambda_{z}^{\prime}(a), \lambda_{z}^{\prime}(b)\right) \mid(a, b) \in \eta\right\}=\left\{\left(\left(\lambda_{z}^{\prime}\right)^{-1}(a),\left(\lambda_{z}^{\prime}\right)^{-1}(b)\right) \mid(a, b) \in \eta\right\}
$$

and $\eta$ also is a congruence on $(M, \circ)$.

Proof. First we shall prove that $\eta^{\prime}$ is a congruence on $(M,+)$. Clearly $\eta^{\prime}$ is reflexive and symmetric because so is each $\eta_{n}$. Let $a, b, c \in M$ such that $(a, b),(b, c) \in \eta^{\prime}$. There exists a positive integer $m$ such that $(a, b),(b, c) \in \eta_{2 m+1}$. Since $\eta_{2 m+1}$ is the transitive closure of $\eta_{2 m}$, we have that $(a, c) \in \eta_{2 m+1} \subseteq \eta^{\prime}$. Hence $\eta^{\prime}$ is an equivalence relation. Note that every $\eta_{n}$ satisfies that $(x+z, y+z) \in \eta_{n}$, for all $(x, y) \in \eta_{n}$. Hence $(a+c, b+c) \in \eta_{2 m+1} \subseteq \eta^{\prime}$. Since $(a, b) \in \eta_{2 m+1}$, we have that $(c+a, c+b) \in \eta_{2 m+2} \subseteq \eta^{\prime}$. Therefore $\eta^{\prime}$ is a congruence.

Let $a, b, c \in M$ be elements such that $(c+a, c+b) \in \eta^{\prime}$. There exists a positive integer $t$ such that $(c+a, c+b) \in \eta_{2 t+1}$. Thus $(a, b) \in \eta_{2 t+2} \subseteq \eta^{\prime}$. Hence $(M,+) / \eta^{\prime}$ is a left cancellative monoid. Since $\eta^{\prime} \subseteq \eta$, we have $\eta^{\prime}=\eta$ by the definition of $\eta$.

Let $(a, b) \in \eta_{0}$. Then there exists $c \in M$ such that $c+a=c+b$. Let $z \in M$. We have that

$$
\left(\lambda_{z}^{\prime}\right)^{\varepsilon}(c)+\left(\lambda_{z}^{\prime}\right)^{\varepsilon}(a)=\left(\lambda_{z}^{\prime}\right)^{\varepsilon}(c+a)=\left(\lambda_{z}^{\prime}\right)^{\varepsilon}(c+b)=\left(\lambda_{z}^{\prime}\right)^{\varepsilon}(c)+\left(\lambda_{z}^{\prime}\right)^{\varepsilon}(b),
$$

for $\varepsilon= \pm 1$. Therefore $\eta_{0}=\left\{\left(\lambda_{z}^{\prime}(a), \lambda_{z}^{\prime}(b)\right) \mid(a, b) \in \eta_{0}\right\}=\left\{\left(\left(\lambda_{z}^{\prime}\right)^{-1}(a),\left(\lambda_{z}^{\prime}\right)^{-1}(b)\right) \mid(a, b) \in \eta_{0}\right\}$. Thus, clearly

$$
\eta_{1}=\left\{\left(\lambda_{z}^{\prime}(a), \lambda_{z}^{\prime}(b)\right) \mid(a, b) \in \eta_{1}\right\}=\left\{\left(\left(\lambda_{z}^{\prime}\right)^{-1}(a),\left(\lambda_{z}^{\prime}\right)^{-1}(b)\right) \mid(a, b) \in \eta_{1}\right\} .
$$

Let $(a, b) \in \eta_{2}$, then either there exist $c, a^{\prime}, b^{\prime} \in M$ such that $\left(a^{\prime}, b^{\prime}\right) \in \eta_{1}$ and $(a, b)=\left(c+a^{\prime}, c+b^{\prime}\right)$, or there exists $d \in M$ such that $(d+a, d+b) \in \eta_{1}$. In the first case, we have that

$$
\left(\left(\lambda_{z}^{\prime}\right)^{\varepsilon}(a),\left(\lambda_{z}^{\prime}\right)^{\varepsilon}(b)\right)=\left(\left(\lambda_{z}^{\prime}\right)^{\varepsilon}(c)+\left(\lambda_{z}^{\prime}\right)^{\varepsilon}\left(a^{\prime}\right),\left(\lambda_{z}^{\prime}\right)^{\varepsilon}(c)+\left(\lambda_{z}^{\prime}\right)^{\varepsilon}\left(b^{\prime}\right)\right)
$$

for $\varepsilon= \pm 1$. Since $\left(\left(\lambda_{z}^{\prime}\right)^{\varepsilon}\left(a^{\prime}\right),\left(\lambda_{z}^{\prime}\right)^{\varepsilon}\left(b^{\prime}\right)\right) \in \eta_{1}$, we get that $\left(\left(\lambda_{z}^{\prime}\right)^{\varepsilon}(a),\left(\lambda_{z}^{\prime}\right)^{\varepsilon}(b)\right) \in \eta_{2}$, in this case. In the second case, since $(d+a, d+b) \in \eta_{1}$, we have that $\left(\left(\lambda_{z}^{\prime}\right)^{\varepsilon}(d)+\left(\lambda_{z}^{\prime}\right)^{\varepsilon}(a),\left(\lambda_{z}^{\prime}\right)^{\varepsilon}(d)+\left(\lambda_{z}^{\prime}\right)^{\varepsilon}(b)\right) \in \eta_{1}$. Thus also in this case we have that $\left(\left(\lambda_{z}^{\prime}\right)^{\varepsilon}(a),\left(\lambda_{z}^{\prime}\right)^{\varepsilon}(b)\right) \in \eta_{2}$. Therefore

$$
\eta_{2}=\left\{\left(\lambda_{z}^{\prime}(a), \lambda_{z}^{\prime}(b)\right) \mid(a, b) \in \eta_{2}\right\}=\left\{\left(\left(\lambda_{z}^{\prime}\right)^{-1}(a),\left(\lambda_{z}^{\prime}\right)^{-1}(b)\right) \mid(a, b) \in \eta_{2}\right\}
$$

Now it is easy to show by induction on $n$ that

$$
\eta_{n}=\left\{\left(\lambda_{z}^{\prime}(a), \lambda_{z}^{\prime}(b)\right) \mid(a, b) \in \eta_{n}\right\}=\left\{\left(\left(\lambda_{z}^{\prime}\right)^{-1}(a),\left(\lambda_{z}^{\prime}\right)^{-1}(b)\right) \mid(a, b) \in \eta_{n}\right\}
$$

for all non-negative integer $n$. Hence

$$
\eta=\left\{\left(\lambda_{z}^{\prime}(a), \lambda_{z}^{\prime}(b)\right) \mid(a, b) \in \eta\right\}=\left\{\left(\left(\lambda_{z}^{\prime}\right)^{-1}(a),\left(\lambda_{z}^{\prime}\right)^{-1}(b)\right) \mid(a, b) \in \eta\right\} .
$$

Let $(a, b) \in \eta_{0}$. Then there exists $c \in M$ such that $c+a=c+b$. Hence $c \circ\left(\lambda_{c}^{\prime}\right)^{-1}(a)=c+a=c+b=$ $c \circ\left(\lambda_{c}^{\prime}\right)^{-1}(b)$. Hence,

and thus

$$
\lambda_{c}^{\prime} \lambda_{\left(\lambda_{c}^{\prime}\right)^{-1}(a)}^{\prime}=\lambda_{c \circ\left(\lambda_{c}^{\prime}\right)^{-1}(a)}^{\prime}=\lambda_{c \circ\left(\lambda_{c}^{\prime}\right)^{-1}(b)}^{\prime}=\lambda_{c}^{\prime} \lambda_{\left(\lambda_{c}^{\prime}\right)^{-1}(b)}^{\prime}
$$

$$
\lambda_{\left(\lambda_{c}^{\prime}\right)^{-1}(a)}^{\prime}=\lambda_{\left(\lambda_{c}^{\prime}\right)^{-1}(b)}^{\prime}
$$

Since $\eta_{0}=\left\{\left(\lambda_{c}^{\prime}(a), \lambda_{c}^{\prime}(b)\right) \mid(a, b) \in \eta_{0}\right\}$, we have that $\lambda_{a}^{\prime}=\lambda_{b}^{\prime}$, for all $(a, b) \in \eta_{0}$. Because

$$
\eta_{n}=\left\{\left(\lambda_{z}^{\prime}(a), \lambda_{z}^{\prime}(b)\right) \mid(a, b) \in \eta_{n}\right\}
$$

for all non-negative integers $n$, it is easy to prove, by induction on $n$, that $\lambda_{a}^{\prime}=\lambda_{b}^{\prime}$, for all $(a, b) \in \eta_{n}$. Hence $\lambda_{a}^{\prime}=\lambda_{b}^{\prime}$, for all $(a, b) \in \eta$. 

have

Let $(a, b) \in \eta$. Then $\left(\lambda_{c}^{\prime}(a), \lambda_{c}^{\prime}(b)\right) \in \eta$. Thus $(c \circ a, c \circ b)=\left(c+\lambda_{c}^{\prime}(a), c+\lambda_{c}^{\prime}(b)\right) \in \eta$. Since $\lambda_{a}^{\prime}=\lambda_{b}^{\prime}$, we

$$
(a \circ c, b \circ c)=\left(a+\lambda_{a}^{\prime}(c), b+\lambda_{b}^{\prime}(c)\right)=\left(a+\lambda_{a}^{\prime}(c), b+\lambda_{a}^{\prime}(c)\right) \in \eta .
$$

Hence $\eta$ is a congruence on $(M, \circ)$, and the result follows.

With the assumptions and notations as in Example $5.2 \quad \bar{M}=M / \eta$. Let $M \longrightarrow \bar{M}: a \mapsto \bar{a}$ be the natural projection. Let $\bar{\lambda}:(\bar{M}, \circ) \longrightarrow \operatorname{Aut}(\bar{M},+)$ be the map defined by $\bar{\lambda}(\bar{a})=\bar{\lambda}_{\bar{a}}$ and $\bar{\lambda}_{\bar{a}}(\bar{b})=\overline{\lambda_{a}^{\prime}(b)}$, for all $a, b \in M$.

Note that $\bar{\lambda}$ is well-defined, because if $\bar{c}=\bar{a}$ and $\bar{d}=\bar{b}$, then, by Lemma 5.5, $\overline{\lambda_{a}^{\prime}(b)}=\overline{\lambda_{a}^{\prime}(d)}$ and $\lambda_{a}^{\prime}=\lambda_{c}^{\prime}$, and

$$
\overline{\lambda_{a}^{\prime}(b)}=\overline{\lambda_{a}^{\prime}(d)}=\overline{\lambda_{c}^{\prime}(d)}
$$

Now it is easy to check that $\bar{\lambda}_{\bar{a}} \in \operatorname{Aut}(\bar{M},+)$ and that $\bar{\lambda}$ is a homomorphism such that $\bar{a} \circ \bar{b}=\bar{a}+\bar{\lambda}_{\bar{a}}(\bar{b})$, for all $a, b \in M$.

Remark 5.6 If, furthermore, the left non-degenerate set-theoretic solution $(X, r)$ is finite and bijective then one can say more. To do so, it is convenient to keep the notation $M=M(X, r)$ and $A=A(X, r)$. So $M \subseteq A \rtimes \operatorname{Im} \lambda^{\prime}$. Jespers, Kubat and Van Antwerpen [19. Proposition 2.9] proved that there exists $t \geq 1$ and a central element $(z, 1) \in M$, with $z \in Z(A)$ and $g(z)=z$ for all $g \in \operatorname{Im}\left(\lambda^{\prime}\right)$, such that the least cancellative congruence on $(A,+)$ is

$$
\begin{aligned}
\eta & =\{(a, b) \in A \times A \mid a+\underbrace{z+\cdots+z}_{\mathrm{i} \text { times }}=b+\underbrace{z+\cdots+z}_{\mathrm{i} \text { times }}, \text { for all } i \geq t\} \\
& =\{(a, b) \in A \times A \mid c+a=c+b \text { for some } c \in A\} \\
& =\eta_{0}
\end{aligned}
$$

Note that $(a, b) \in \eta$ implies that $\lambda_{a}^{\prime}=\lambda_{b}^{\prime}$. Hence, it follows from Proposition 4.2 in [19] that the (least) cancellative congruence on $(M, \circ)$ is

$$
\eta_{M}=\left\{\left(\left(a, \lambda_{a}^{\prime}\right),\left(b, \lambda_{b}^{\prime}\right)\right) \mid(a, b) \in \eta\right\} .
$$

It follows that the natural map

$$
M / \eta_{M} \longrightarrow(A / \eta) \rtimes \operatorname{Im}\left(\lambda^{\prime}\right)
$$

i.e. $\overline{\left(a, \lambda_{a}^{\prime}\right)} \mapsto\left(\bar{a}, \lambda_{a}^{\prime}\right)$, is an injective monoid homomorphism and $M / \eta_{M}$ is a regular submonoid of $(A / \eta) \rtimes$ $\operatorname{Im}\left(\lambda^{\prime}\right)$. So we obtain a bijective 1 -cocycle $\left(M / \eta_{M}, \circ\right) \longrightarrow(A / \eta,+)$, with respect to $\bar{\lambda}$, that extends the mapping $\overline{\left(a, \lambda_{a}^{\prime}\right)} \mapsto \bar{a}$. Because $r$ is bijective we know (see explanation in Example 5.2 ) that $(A,+)$ consists of normal elements and thus $(A / \eta,+)$ is a left and right Ore monoid and also $\left(M / \eta_{M}, \circ\right)$ is a left and right Ore monoid. Hence they both have a group of fractions, denoted $\operatorname{gr}(A / \eta)$ and $\operatorname{gr}\left(\left(M / \eta_{M}\right)\right.$ respectively. It easily is verified that $\operatorname{gr}\left(M / \eta_{M}\right)=G(X, r)$, the structure group of $(X, r), \operatorname{gr}(A / \eta)=G\left(X, r^{\prime}\right)$, the structure group of the derived solution $\left(X, r^{\prime}\right), \operatorname{gr}\left(M / \eta_{M}\right) \subseteq \operatorname{gr}(A / \eta) \rtimes \operatorname{Im}\left(\lambda^{\prime}\right)$, where by abuse of notation $\lambda^{\prime}: \operatorname{gr}\left(M / \eta_{M}\right) \rightarrow \operatorname{Aut}(A / \eta)$ is the natural extension of the mapping $\bar{\lambda}$ and also $\operatorname{gr}\left(M / \eta_{M}\right)$ is a regular subgroup of $\operatorname{gr}(A / \eta) \rtimes \operatorname{Im}\left(\lambda^{\prime}\right)$. The latter was proven by Lebed and Vendramin in [24, Theorem 3.4.] in case $(X, r)$ is bijective, (left and right) non-degenerate and finite.

Question 5.7 If $(X, r)$ is a left non-degenerate solution of the YBE does there exist a bijective 1-cocycle $\left(M / \eta_{M}, \circ\right) \longrightarrow(A / \eta,+)$, with respect to $\bar{\lambda}$, that extends the mapping $\overline{\left(a, \lambda_{a}^{\prime}\right)} \mapsto \bar{a}$. In other words, can one avoid the bijective assumption in Remark [5.6]?

Let $\bar{\phi}: \bar{M} \times \bar{M} \longrightarrow \bar{M}$ be the map defined by $\bar{\phi}(\bar{a}, \bar{b})=\bar{\lambda}_{\bar{a}}(\bar{b})$, for all $a, b \in M$. Then $(\bar{M},+, \circ, \bar{\phi})$ is a left semi-truss.

Lemma 5.8 Let $a, b \in M=M(X, r)$. Then there exists $c \in M$ such that $a+b=b+c$. 
Proof. There exist non-negative integers $n, m$ and $x_{1}, \ldots, x_{n}, y_{1}, \ldots, y_{m} \in X$ such that $a=x_{1}+\cdots+x_{n}$ and $b=y_{1}+\cdots+y_{m}$. Clearly we may assume that $n, m$ are positive integers. We shall prove the result by induction on $n+m$. If $n=m=1$, then $x_{1}+y_{1}=y_{1}+\sigma_{y_{1}}\left(\gamma_{\sigma_{x_{1}}^{-1}\left(y_{1}\right)}\left(x_{1}\right)\right)$, by the defining relations of $(M,+)$. Suppose that $m+n>2$, and that the result is true for $m+n-1$. If $n>1$, then by the induction hypothesis there exists $c^{\prime} \in M$ such that $a+b=x_{1}+b+c^{\prime}$, and by the induction hypothesis again there exists $c^{\prime \prime} \in M$ such that $x_{1}+b=b+c^{\prime \prime}$. Hence $a+b=b+c^{\prime \prime}+c^{\prime}$, in this case. Suppose that $n=1$. In this case $m>1$ and

$$
a+b=x_{1}+b=y_{1}+\sigma_{y_{1}}\left(\gamma_{\sigma_{x_{1}}^{-1}\left(y_{1}\right)}\left(x_{1}\right)\right)+y_{2}+\cdots+y_{m}
$$

Hence, by the induction hypothesis, there exists $c \in M$ such that

$$
\sigma_{y_{1}}\left(\gamma_{\sigma_{x_{1}}^{-1}\left(y_{1}\right)}\left(x_{1}\right)\right)+y_{2}+\cdots+y_{m}=y_{2}+\cdots+y_{m}+c .
$$

Thus $a+b=b+c$, in this case. Therefore the result follows by induction.

By Lemma 5.8, the left cancellative monoid $(\bar{M},+)$ satisfies that for all $\bar{a}, \bar{b} \in \bar{M}$, there exists a unique $\bar{c} \in \bar{M}$ such that $\bar{a}+\bar{b}=\bar{b}+\bar{c}$. So, the multiplicative monoid $(\bar{M}, \circ)$ is left cancellative. Hence, we have the following corollary.

Corollary 5.9 Let $(X, r)$ be a left non-degenerate set-theoretic solution of the YBE. Let $\eta$ be the left cancellative congruence on $\left(M\left(X, r^{\prime}\right),+\right)$. Then $(\bar{M},+, \circ, \bar{\phi})$ is a left semi-truss with $\bar{M}+\bar{a} \subseteq \bar{a}+\bar{M}$ for all $\bar{a} \in \bar{M}$ and it satisfies the conditions of Proposition 5.4 , with $\bar{\phi}(\bar{a}, \bar{b})=\bar{\lambda}_{\bar{a}}(\bar{b})$, for all $\bar{a}, \bar{b} \in \overline{\bar{M}}$. In particular, $(\bar{M}, \bar{r})$, where

$$
\bar{r}(\bar{a}, \bar{b})=\left(\bar{\lambda}_{\bar{a}}(\bar{b}), \bar{\lambda}_{\bar{\lambda}_{\bar{a}(\bar{b})}^{-1}}\left(c\left(\bar{a}, \bar{\lambda}_{\bar{a}}(\bar{b})\right)\right)\right)
$$

for all $\bar{a}, \bar{b} \in \bar{M}$, is a left non-degenerate set-theoretic solution of the YBE. In particular, $\left(\bar{X}, \bar{r}_{\mid \bar{X}}\right)$ is a left non-degenerate solution on the image $\bar{X}$ of $X$ in $\bar{M}$.

We say that a left non-degenerate solution $(X, r)$ of the YBE is injective if the natural map $X \rightarrow M / \eta$ is injective. Obvious such examples are irretractable solutions, and in this case $r=\left.\bar{r}\right|_{\bar{X}^{2}}$. Note that if $r$ also is bijective and non-degenerate then this notion corresponds with the one introduced by Lebed and Vendramin in [24]. In 24] it also is shown that, in this case, several properties of involutive solutions can be generalized to injective ones.

Corollary 5.10 Any left non-degenerate injective set-theoretic solution $(X, r)$ of the YBE is the restriction of the induced left-non-degenerate solution of the YBE determined by a left cancellative semi-truss $(M,+, \circ, \phi)$ with $M+a \subseteq a+M$ for all $a \in M$.

However, note that $(\bar{M}, \circ)$ is not necessarily the structure monoid of the solution of $(\bar{X}, \bar{r})$. Indeed, let $X=\mathrm{Sym}_{3}$ be the symmetric group of degree 3 . Let $(X, r)$ be the bijective non-degenerate solution defined by $r(a, b)=\left(a b a^{-1}, a\right)$ for all $a, b \in X$. Note that the solution $(X, r)$ is non-involutive and irretractable (because the center of $\operatorname{Sym}_{3}$ is trivial). So, $X$ is naturally embedded in $(\bar{M}, \circ)=(M(X, r) / \eta, \circ)$ and $\bar{r}_{\mid \bar{X}^{2}}=r$. Let us denote the multiplication in the structure monoid $M(X, r)$ by $\cdot$ In $(M(X, r), \cdot)$ we have

$$
\begin{aligned}
(1,2) \cdot(1,2,3) \cdot(1,2,3) \cdot(1,2,3) & =(1,3,2) \cdot(1,3,2) \cdot(1,3,2) \cdot(1,2) \\
& =(1,3,2) \cdot(1,3,2) \cdot(1,3) \cdot(1,3,2) \\
& =(1,3,2) \cdot(2,3) \cdot(1,3,2) \cdot(1,3,2) \\
& =(1,2) \cdot(1,3,2) \cdot(1,3,2) \cdot(1,3,2)
\end{aligned}
$$

while $(1,2,3) \cdot(1,2,3) \cdot(1,2,3) \neq(1,3,2) \cdot(1,3,2) \cdot(1,3,2)$. Hence, $M(X, r)$ is not left cancellative, while $\bar{M}$ is left cancellative. Thus $\bar{M}$ is not the structure monoid of $(X, r)$.

The following problem remains a challenge. 
Question 5.11 Determine when a left non-degenerate solution $(X, r)$ of the $Y B E$ is cancellative injective. If $(X, r)$ is a left non-degenerate solution that is injective then does there exists a finite left cancellative semi-truss in which $X$ can be embedded naturally? In case $r$ also is finite, bijective and non-degenerate this has been proven by Lebed and Vendramin in [24].

Acknowledgement. The authors would like to thank the referee for providing a sub- and superscript avoiding, more elegant and simple proof of Theorem 4.5 by using the notation and a result of Rump proved in $[29$.

\section{References}

[1] D. Bachiller, Solutions of the Yang-Baxter equation associated to skew left braces, with applications to racks, J. Knot Theory Ramifications 27 (2018), no. 8, 1850055, 36 pp.

[2] D. Bachiller, F. Cedó, and E. Jespers, Solutions of the Yang-Baxter equation associated with a left brace, J. Algebra 463 (2016), 80-102.

[3] D. Bachiller, F. Cedó, and L. Vendramin, A characterization of finite multipermutation solutions of the Yang-Baxter equation, Publ. Mat., 62(2) (2018), 641-649.

[4] T. Brzeziński, Towards semi-trusses, Rev. Roumaine Math. Pures Appl. 63 (2018), no. 2, 75-89.

[5] F. Cedó, E. Jespers, and J. Okniński, Braces and the Yang-Baxter equation, Comm. Math. Phys. 327(1) (2014), 101-116.

[6] F. Cedó and J. Okniński, Gröbner bases for quadratic algebras of skew type, Proc. Edinb. Math. Soc. (2) 55 (2012), no. 2, 387-401.

[7] F. Chouraqui, Garside groups and Yang-Baxter equation, Comm. Algebra, 38(12) (2010), 4441-4460.

[8] F. Chouraqui, Left orders in Garside groups, Internat. J. Algebra Comput., 26(7) (2016), 1349-1359.

[9] P. Dehornoy, Set-theoretic solutions of the Yang-Baxter equation, RC-calculus, and Garside germs. Adv. Math., 282 (2015), 93-127.

[10] K. Cvetko-Vah and C. Verwimp, Skew lattices and set-theoretic solutions of the Yang-Baxter equation, J. Algebra 542 (2020), 65-92.

[11] V.G. Drinfeld, On some unsolved problems in quantum group theory, in: P.P. Kulish (Ed.), Quantum Groups, in:Lecture Notes in Math., vol. 1510, Springer-Verlag, 1992, pp. 1-8.

[12] P. Etingof, T. Schedler, and A. Soloviev, Set-theoretical solutions to the quantum Yang-Baxter equation, Duke Math. J. 100(2) (1999), 169-209.

[13] T. Gateva-Ivanova, Quadratic algebras, Yang-Baxter equation, and Artin-Schelter regularity, Adv. Math., 230(4-6) (2012), 2152-2175.

[14] T. Gateva-Ivanova and P. Cameron, Multipermutation solutions of the Yang-Baxter equation, Comm. Math. Phys. 309(3) (2012), 583-621.

[15] T. Gateva-Ivanova, E. Jespers and J. Okniński, Quadratic algebras of skew type and the underlying monoids, J. Algebra 270 (2003), no. 2, 635-659.

[16] T. Gateva-Ivanova and S. Majid, Matched pairs approach to set-theoretic solutions of the Yang-Baxter equation, J. Algebra 319 (2008), no. 4, 1462-1529.

[17] T. Gateva-Ivanova and M. Van den Bergh, Semigroups of I-type, J. Algebra 206(1) (1998), 97-112.

[18] L. Guarnieri and L. Vendramin, Skew braces and the Yang-Baxter equation, Math. Comp. 86 (2017), no. 307, 2519-2534.

[19] E. Jespers, L. Kubat, and A. Van Antwerpen, The structure monoid and algebra of a non-degenerate set-theoretic solution of the Yang-Baxter equation, Trans. Amer. Soc. 372 (2019), no. 10, 7191-7223. Corrigendum and addendum to "The structure monoid and algebra of a non-degenerate set-theoretic solution of the Yang-Baxter equation". Trans. Amer. Math. Soc. 373 (2020), no. 6, 4517-4521.

[20] E. Jespers and J. Okniński, Monoids of $I$-type, Algebr. Represent. Theory 8 (2005), no. 5, 709-729. 
[21] E. Jespers and J. Okniński, Noetherian semigroup algebras, volume 7 of Algebras and Applications, Springer, Dordrecht, 2007.

[22] E. Jespers and M. Van Campenhout, Finitely generated algebras defined by homogeneous quadratic monomial relations and their underlying monoids II, J. Algebra 492 (2017), 524-546.

[23] E. Jespers, J. Okniński and M. Van Campenhout, Finitely generated algebras defined by homogeneous quadratic monomial relations and their underlying monoids, J. Algebra 440 (2015), 72-99.

[24] V. Lebed and L. Vendramin, On structure groups of set-theoretic solutions to the Yang-Baxter equation, Proc. Edinb. Math. Soc. (2) 62 (2019), no. 3, 683-717.

[25] J.-H. Lu, M. Yan, and Y.-C. Zhu, On the set-theoretical Yang-Baxter equation, Duke Math. J., 104(1) (2000), 1-18.

[26] W. Rump, A decomposition theorem for square-free unitary solutions of the quantum Yang-Baxter equation, Adv. Math. 193(1) (2005), 40-55.

[27] W. Rump, Braces, radical rings, and the quantum Yang-Baxter equation, J. Algebra, 307(1) (2007), $153-170$.

[28] W. Rump, The brace of a classical group. Note Mat., 34(1) (2014), 115-144.

[29] W. Rump, A covering theory for non-involutive set-theoretic solutions to the Yang-Baxter equation, J. Algebra, 520 (2019), 136-70.

[30] A. Smoktunowicz and L. Vendramin, On skew braces (with an appendix by N. Byott and L. Vendramin), J. Comb. Algebra, 2(1) (2018), 47-86.

[31] A. Soloviev, Non-unitary set-theoretical solutions to the quantum Yang-Baxter equation, Math. Res. Lett. 7 (2000), 577-596.

[32] M. Takeuchi, Survey on matched pairs of groups-an elementary approach to the ESS-LYZ theory, in Noncommutative geometry and quantum groups (Warsaw, 2001), volume 61 of Banach Center Publ., pages 305-331. Polish Acad. Sci., Warsaw, 2003.

[33] A. Weinstein and P. Xu, Classical solutions of the quantum Yang-Baxter equation, Comm. Math. Phys. 148(2) (1992), 309-343.

\author{
Ferran Cedó \\ Departament de Matemàtiques \\ Universitat Autònoma de Barcelona \\ 08193 Bellaterra (Barcelona), Spain \\ cedo@mat.uab.cat \\ Charlotte Vermwimp \\ Department of Mathematics \\ Vrije Universiteit Brussel \\ Pleinlaan 2, 1050 Brussel, Belgium \\ Charlotte.Verwimp@vub.be
}

\author{
Eric Jespers \\ Department of Mathematics \\ Vrije Universiteit Brussel \\ Pleinlaan 2, 1050 Brussel, Belgium \\ Eric.Jespers@vub.be
}

